\title{
Convergence in Probability of Compressed Annealing
}

Jeff Ohlmann

\author{
James Bean
}

\author{
Shane Henderson
}

\begin{abstract}
We consider combinatorial optimization problems for which the formation of a neighborhood structure of feasible solutions is impeded by a set of constraints. Neighborhoods are recovered by relaxing the complicating constraints into the objective function within a penalty term. We examine a heuristic called compressed annealing that integrates a variable penalty multiplier approach within the framework of simulated annealing. We refer to the value of the penalty multiplier as "pressure." We analyze the behavior of compressed annealing by exploring the interaction between temperature (which controls the ability of compressed annealing to climb hills) and pressure (which controls the height of the hills). We develop a necessary and sufficient condition on the joint cooling and compression schedules for compressed annealing to converge in probability to the set of global minima. Our work generalizes the results of Hajek (1988) in the sense that when there are no relaxed constraints, our results reduce to his.
\end{abstract}

Keywords: simulated annealing, penalty methods, constrained optimization.

\section{Introduction}

A combinatorial optimization problem can be formulated as:

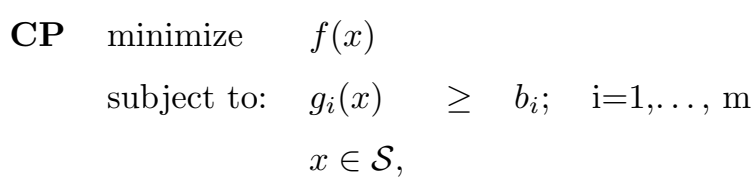

where $\mathcal{S}$ is a finite set, and $f(\cdot)$ and $g_{i}(\cdot)$ are real-valued functions on $\mathcal{S}$. We are particularly interested in instances of $\mathbf{C P}$ classified as NP-hard. The intractability of these problems suggests the application of metaheuristics to find near-optimal solutions. A trait common to many metaheuristic approaches is the requirement of "neighborhood" structures to generate candidate solutions from a current solution (Reeves, 1993). We consider instances of CP for which the formation of a neighborhood structure of feasible solutions is impeded by the constraints $\left\{g_{i}(x) \geq b_{i}\right\}$ for $i=1, \ldots, m$. We recover well-defined neighborhoods by relaxing the complicating constraints into the objective function with a penalty term.

\subsection{Penalty Methods and Annealing}

For a solution $x \in \mathcal{S}$, let $p(x)$ be a real-valued, nonnegative function indicating violation of the constraints $\left\{g_{i}(x) \geq b_{i}\right\}$ for $i=1, \ldots, m$, so that $p(x)>0$ if and only if $x$ is infeasible. We let $\lambda$ be a nonnegative, scalar penalty multiplier, and refer to the function $v(x, \lambda)=f(x)+\lambda p(x)$ as the auxiliary function. Then we formulate $\mathbf{R P}(\lambda)$, a relaxation of $\mathbf{C P}$ as 


$$
\begin{array}{rll}
\mathbf{R P}(\lambda) & \text { minimize } & v(x, \lambda)=f(x)+\lambda p(x) \\
& \text { subject to: } & x \in \mathcal{S}
\end{array}
$$

We focus on solving $\mathbf{C P}$ via an implementation of simulated annealing on $\mathbf{R P}(\lambda)$. Simulated annealing is a stochastic search method in which the ability to "climb hills" is governed by a control parameter called temperature. Since the introduction of simulated annealing by Kirkpatrick et al. (1983) and Cerny (1985) in independent efforts based on the Metropolis algorithm (Metropolis et al., 1953), there has been a multitude of research on various aspects of the algorithm. Of particular concern is the manner in which the temperature parameter is manipulated. Complying with the analogy of physical annealing, temperature traditionally is initiated at a high value (corresponding to a high probability of accepting transitions to nonimproving solutions) and slowly cooled to zero. For high temperatures, simulated annealing is essentially a form of random search, which suffers from the curse of dimensionality. At the other extreme, as temperature approaches zero, simulated annealing becomes a descent method and cannot escape local minima. Thus, to capture the benefits of being able to escape local minima and still satisfactorily explore the basins containing them, a reasonable approach is to reduce the temperature as the algorithm progresses.

The rate at which the temperature parameter is reduced is a topic of both theoretical and empirical interest. Geman and Geman (1984), Anily and Federgruen (1987), Mitra et al. (1986), and Johnson and Jacobson (2002) determine various sufficient conditions on the cooling schedule for convergence in probability to a global minimum. Chiang and Chow (1988) and Holley and Stroock (1988) also provide convergence results. Lundy and Mees (1986) suggest a cooling schedule and stopping condition designed to produce a solution within $\epsilon$ of the global optimum with a given probability. Using results from continuous-time Markov chains, Gidas (1985) and Hajek (1988) present cooling schedules which satisfy necessary and sufficient conditions for convergence in probability to the set of global minima. This research on the theoretical behavior of simulated annealing provided insight on its implementation and sparked the proliferation of many variants.

To solve $\mathbf{R P}(\lambda)$, we have an additional parameter to consider, namely the value of the penalty multiplier $\lambda$. Maintaining the physical analogy of simulated annealing, we call this parameter "pressure" (Morse, 1997). The set $\mathcal{S}$ is finite, so for sufficiently large $\lambda$, any optimal solution to the relaxation $\mathbf{R P}(\lambda)$ is optimal for CP (we call this property strong duality); see Hadj-Alouane and Bean (1997). Unfortunately, for large-scale problems, it is impractical to determine the exact multiplier value at which strong duality first holds. Fixing pressure at a "large" value to avoid converging to infeasible solutions might seem reasonable, but this makes it difficult for the annealing algorithm to move through the solution space. The high penalties mean that infeasible solutions are excessively penalized, and so practically speaking, the search is limited to feasible solutions. Fixing pressure at a "small" value ensures that the annealing algorithm can more easily move through the solution space, but one could converge to an infeasible point. In view of these observations, and because computational experience has demonstrated that it is often difficult to determine a "good" value for pressure, we examine a heuristic called compressed annealing (Morse, 1997). Compressed annealing 
simultaneously adjusts pressure and temperature within the annealing run. In this paper, we will confine our attention to temperature schedules that are decreasing, and pressure schedules that are increasing.

Similar constrained annealing approaches have been explored in the literature. Aarts and Korst (1989) examine simulated annealing in the context of several constrained combinatorial problems. Geman (1990) provides convergence results for the application of the Gibbs sampler on an appropriately conditioned state space. Using Dobrushin's contraction technique, Yao (2000) extends the work of Geman (1990) to provide a sufficient condition on the convergence of constrained simulated annealing. Robini et al. (2002) improves this sufficient condition by providing a tight upper bound on the second largest eigenvalue in absolute value of the transition probability matrix associated with the underlying Metropolis chain. Frigerio and Grillo (1993) and Del Moral and Miclo (1999) also consider annealing with time-depended energy functions, but under assumptions that imply an upper bound on the penalty multiplier.

\subsection{Motivation of Our Results}

Ideally, one would perhaps like to construct cooling and compression schedules that minimize the expected time required to find a global optimum. A first-order concern related to this goal is whether the time required to find a global minimum is almost-surely finite or not. In Theorem 3, we show that if the sum of the expected jump probabilities, denoted $\sum_{k=0}^{\infty} \eta\left(d_{k}\right)$, is finite, then there is a positive probability that a global minimum will never be reached (from certain states), i.e., the time to hit a global minimum, $T_{\text {global }}$, is infinite. We can conclude that if $P\left\{T_{\text {global }}=\infty\right\}=0$, then $\sum_{k=0}^{\infty} \eta\left(d_{k}\right)=\infty$.

We also prove the converse, i.e., if $\sum_{k=0}^{\infty} \eta\left(d_{k}\right)=\infty$, then $P\left\{T_{\text {global }}=\infty\right\}=0$, by supplying a stronger converse. Specifically, in Theorem 1 we show that if $\sum_{k=0}^{\infty} \eta\left(d_{k}\right)=\infty$, then $\lim _{k \rightarrow \infty} P\left\{Y(k) \in \mathcal{G}^{*}\right\}=1$, where $\mathcal{G}^{*}$ is the set of global minima.

So the main result of this paper is a set of necessary and sufficient conditions for compressed annealing to converge in probability to a global minimum. This result is a generalization of that in Hajek (1988), in the sense that when there are no relaxed constraints, our results reduce to his. Our proof of this result follows a path hewn by Hajek, although many aspects of the proof involve nontrivial extensions of Hajek's concepts and results. We chose to adopt Hajek's framework as the basis for our approach because his necessary and sufficient conditions are the strongest in the literature.

Hajek showed that cooling rates that ensure convergence depend on the shape of the auxiliary function, and in particular, the "depth" of the deepest local, nonglobal minimum. This observation is of particular prominence in our work, where we alter the shape of the auxiliary function via compression during the annealing process. Continued compression deepens the "valleys" and therefore makes it increasingly more difficult for the process to escape local minima. Nevertheless, we prove that even if we allow pressure to increase without bound, there still exists a temperature schedule such that the annealing algorithm converges in probability to the set of global minima. 
In $§ 3.3 .1$, we show that the temperature schedule must converge to 0 slower than $O\left([\ln k]^{-1}\right)$. Therefore, practically speaking, one cannot expect to use compressed annealing to hit the set of global minima with probability 1. One might then consider the less-lofty goal of minimizing the expected time to hit a set of $\epsilon$-optimal solutions, $E\left[T_{\epsilon}\right]$. But from Theorem 3, one can again conclude for some topologies and starting states, if $\sum_{k=0}^{\infty} \eta\left(d_{k}\right)<\infty$, then $P\left\{T_{\epsilon}=\infty\right\}>0$.

Nevertheless, compressed annealing has exhibited success in obtaining reasonable solutions to problems that genetic algorithms and traditional simulated annealing have not (Morse, 1997). Theodoracatos and Grimsley (1995) implement a similar approach using a variable penalty method and simulated annealing to solve a two-dimensional packing problem.

This paper is organized as follows. In $\S 2$, we introduce some basic definitions and describe pressure's effect on the problem structure. We present a Markov chain representation of compressed annealing and its associated terminology in $\S 3$. We conclude $\S 3$ with our main result, its implications, and the outline of the proof. The remainder of the paper is then dedicated to the proof of the main result. In particular, $\S 4$ proves a result quantifying compressed annealing's ability to climb out of local, nonglobal minima. Then $\S 5$ lower-bounds the probability of the process being trapped near a local minima. In $\S 6$, we utilize the results of $\S 4$ and $\S 5$ to state a sufficient condition for the process to settle onto states "near" sufficiently deep local minima. Finally, $\S 7$ completes the proof of the main result.

\section{Dynamics}

In this section, we describe the dynamic behavior induced by the variable multiplier approach. For each state $x \in \mathcal{S}$, define $N(x) \subseteq \mathcal{S}$ as the static neighborhood of $x$, and let $\Gamma$ be a stochastic matrix for generating neighbors.

Assumption 1. For all $x, y \in \mathcal{S}$, such that $y \in N(x)$, there exists a constant $c_{0}$ such that $0<c_{0} \leq \Gamma(x, y) \leq$ 1 , and furthermore, $\Gamma(x, y)>0$ if and only if $y \in N(x)$.

Every state $x \in \mathcal{S}$ is described by the ordered pair $(f(x), p(x))$ consisting of the state's cost and degree of infeasibility. We say that a state $x$ with $f(x)=f$ and $p(x)=p$ is at level $(f, p)$. Levels are ordered such that $(f, p) \succ\left(f^{\prime}, p^{\prime}\right)$ if and only if either (i) $p>p^{\prime}$ or (ii) $p=p^{\prime}$ and $f>f^{\prime}$. Furthermore, $(f, p)=\left(f^{\prime}, p^{\prime}\right)$ if and only if $p=p^{\prime}$ and $f=f^{\prime}$. In this manner, we lexicographically order the states in $\mathcal{S}$ with respect to $p(\cdot)$ and then $f(\cdot)$.

Define a solution topology, $\Theta=(\mathcal{S}, N, f, p)$, as the collection of states in a solution space connected by a neighborhood structure together with the functions $f$ and $p$. A solution topology encapsulates all the essential problem parameters. We also define a solution topography, $\Theta_{\lambda}=(\mathcal{S}, N, v(\cdot, \lambda))$. The topography, $\Theta_{\lambda}$, utilizes the information contained in the topology, $\Theta$, to describe the relational behavior between states for a particular value of $\lambda$. While the topology is static, observe that the topography captures the dynamic 

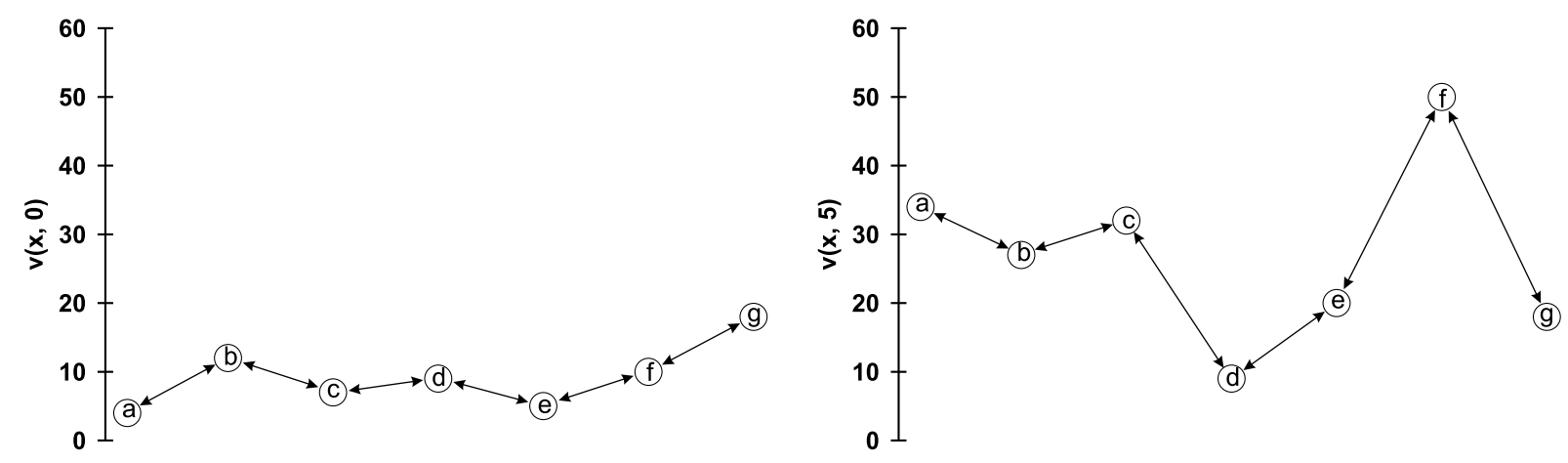

Figure 1: The two diagrams illustrate the dynamic nature of a solution topography for $\lambda=0$ and $\lambda=5$. States $d$ and $g$ are feasible $(p(d)=p(g)=0)$ and the other states are infeasible to varying degrees $(p(f)>$ $p(a)>p(c)>p(b)>p(c)>p(e))$. For this example, $\lambda^{*}=4 . \overline{3}$, so the topography on the left is "volatile" and the topography on the right is "stable."

impact of pressure since a change in $\lambda$ alters the value of the auxiliary function, $v(x, \lambda)$, for every $x$ such that $p(x)>0$.

The ordering of states with respect to $v(\cdot, \lambda)$ may vary with $\lambda$. However, since $|\mathcal{S}|<\infty$, for $\lambda$ sufficiently large, a constant ordering within the solution topography can be attained. Lemma 1 articulates this intuition and its corollary motivates the utilization of $\mathbf{R P}(\lambda)$ to solve $\mathbf{C P}$. The proofs of these results are straightforward and omitted.

Lemma 1. For a solution topography $\Theta_{\lambda}$ with $|\mathcal{S}|<\infty$, the following are true.

(a) There exists $\lambda_{x, y}^{*} \geq 0$ such that if $\lambda>\lambda_{x, y}^{*}$ and $(f(x), p(x)) \succ(f(y), p(y))$, then $v(x, \lambda)>v(y, \lambda)$.

(b) There exists $\lambda^{*} \geq 0$ such that $\lambda>\lambda^{*}$ if and only if $v(x, \lambda)>v(y, \lambda)$ for every pair of states $x, y$ with $(f(x), p(x)) \succ(f(y), p(y))$.

(c) For $\lambda>\lambda^{*}$, all states that are local minima for the original problem $\mathbf{C P}$ are local minima for $\mathbf{R P}(\lambda)$. (Note: Local minima for $\mathbf{R P}(\lambda)$ are not necessarily feasible states.)

Corollary 1. If $x$ is a global minimum of $\mathbf{C P}$, then $x$ is also a global minimum of $\mathbf{R P}(\lambda)$ for $\lambda>\lambda^{*}$.

For $\lambda<\lambda^{*}$, there is a transient period in which the topography is "volatile." That is, the ordering of the states' auxiliary function values relative to each other is not fixed. For $\lambda>\lambda^{*}$, the topography stabilizes in the sense that the ordering of states with respect to $v(\cdot, \lambda)$ agrees with the relation $\succ$. Note that while a constant ordering of states in $\mathcal{S}$ is achieved for all $\lambda>\lambda^{*}$, further compression will increase the difference in the auxiliary function values between any particular pair of states $x$ and $y$ if $p(x)>p(y)$. Figure 1 illustrates the dynamic solution topography in a small example. 


\section{Markov Chain Model and Vernacular}

To begin our analysis, we introduce notation related to the control parameters, temperature and pressure. Define the cooling schedule to be a deterministic, decreasing sequence of strictly positive numbers $\left(\tau_{0}, \tau_{1}, \ldots\right)$ such that $\lim _{t \rightarrow \infty} \tau_{t}=0$. Additionally, define the compression schedule as a deterministic, increasing sequence of nonnegative numbers $\left(\lambda_{0}, \lambda_{1}, \ldots\right)$ such that $\lim _{t \rightarrow \infty} \lambda_{t}=\infty$.

Using the compression schedule, we parameterize the auxiliary function of $\mathbf{R P}(\lambda)$, by defining $v_{t}(x)=$ $f(x)+\lambda_{t} p(x)$. By Lemma 1 and our definition of a compression schedule, there exists $t^{*}<\infty$ such that $\lambda_{t} \leq \lambda^{*}$ for $0 \leq t \leq t^{*}$, and $\lambda_{t}>\lambda^{*}$ for all $t>t^{*}$. That is, $t^{*}$ is the point in the compression schedule at which the topography stabilizes.

We model the compressed annealing algorithm as a time-inhomogeneous, discrete-time Markov chain $(Y(k), T(k): k \geq 0)$ with state space $\mathcal{S} \times\{0,1, \ldots\}$. At step $k$, the current solution of the algorithm is $Y(k)=x$, and $T(k)$ is interpreted as the "clock time." At each step of the algorithm, a candidate solution, $y$, is generated from the mass function, $\Gamma(x, \cdot)$, and $y$ is then accepted with a probability dependent on $\tau_{T(k)}$ and the quantity $h_{T(k)}=\left(v_{T(k)}(y)-v_{T(k)}(x)\right)^{+}$. If $y$ is accepted, then $Y(k+1)=y$, otherwise $Y(k+1)=x$. In $\S 3.1$, we explain how $(T(k): k \geq 0)$ evolves.

Let the acceptance function, $\left(\eta_{t}: t \geq 0\right)$, be a function of temperature, $\tau_{t}$, such that $0<\eta_{t}<1, \eta_{t}$ is nonincreasing in $t$, and $\lim _{t \rightarrow \infty} \eta_{t}=0$. Typically, $\eta_{t}=\exp \left(-1 / \tau_{t}\right)$. This function governs the probability of accepting transitions in our probabilistic hill-climbing algorithm. The term $\eta_{T(k)}^{h_{T(k)}}$ represents the probability of, at step $k$, accepting a transition from the current state, $x$, to a state $y \in N(x)$. Note that the height of the jump between states, $h_{T(k)}=\left\{f(y)-f(x)+\lambda_{T(k)}[p(y)-p(x)]\right\}^{+}$is a function of the penalty multiplier, thus exhibiting the acceptance probability's relationship with the pressure parameter. For notational simplicity, we henceforth write $\eta_{t}^{h_{t}}$ as $\eta\left(h_{t}\right)$.

\subsection{Structuring the Solution Space}

Motivated by stabilization of the solution topography, $\Theta_{\lambda}$, for $\lambda>\lambda^{*}$, we partition $\mathcal{S}$ into sets based on each state's level. Let $L(f, p)=\{x \in \mathcal{S}: f(x)=f, p(x)=p\}$ be the set of states at level $(f, p)$. Note that for any $x \in L(f, p), v_{t}(x)=f+\lambda_{t} p$, i.e., for any value of $\lambda$, all states in the same level have the same auxiliary function value. The order relation $\succ$ partitions $\mathcal{S}$ into $\left\{L\left(f_{1}, p_{1}\right), L\left(f_{2}, p_{2}\right), \ldots, L\left(f_{\ell}, p_{\ell}\right)\right\}$ where $\left(f_{1}, p_{1}\right) \prec\left(f_{2}, p_{2}\right) \prec \cdots \prec\left(f_{\ell}, p_{\ell}\right)$, and $\ell$ is the number of levels in $\mathcal{S}$.

As in Hajek (1988), we assume that the problem is structured so that the process can only climb up one level at a time. This concept is formalized below.

Assumption 2. The topology $\Theta$ possesses the continuous increase property. That is, if $x \in L\left(f_{i}, p_{i}\right)$ and $y \in L\left(f_{j}, p_{j}\right)$ with $j>i+1$, then $y \notin N(x)$.

If a problem's topology possesses the continuous increase property, then for $x \in L\left(f_{i}, p_{i}\right), N(x)$ consists 

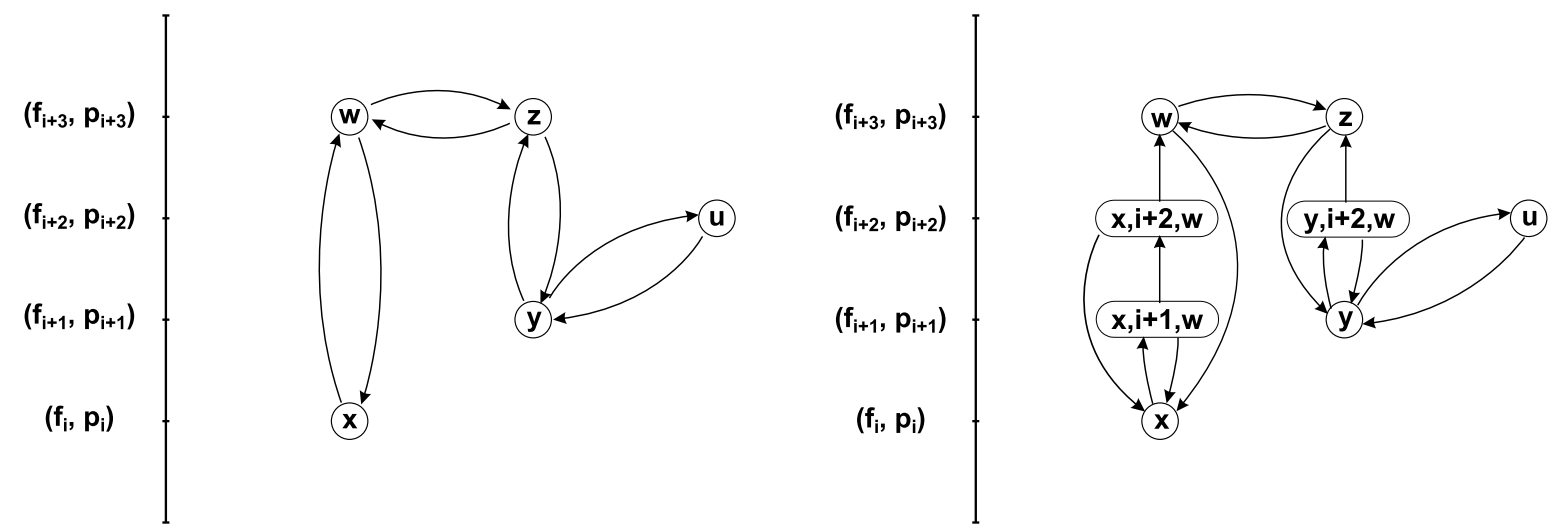

Figure 2: Solution topography (for $\lambda>\lambda^{*}$ ) before and after state augmentation.

only of states $y$ such that $y \in L\left(f_{j}, p_{j}\right)$ where $j \leq i+1$. To interpret this, consider $Y(k)=x \in L\left(f_{i}, p_{i}\right)$ at iteration $k$ where $\lambda_{T(k)}>\lambda^{*}$. Then the "distance" of any uphill transition is $f_{i+1}-f_{i}+\lambda_{T(k)}\left(p_{i+1}-p_{i}\right)$ (since we only climb one level at a time). Thus, for $\lambda$ sufficiently large, the probability of accepting an uphill transition is the same for every state on a given level. Note that this property only affects uphill transitions and not downhill transitions that are always accepted.

At first glance, the continuous increase property appears to be quite restrictive. Hajek (1988) shows that one can introduce artificial states to recover the continuous increase property for the case where all states are feasible. A similar augmentation technique can be applied to our situation.

Suppose that the topology, $\Theta$, does not possess the continuous increase property. The idea is to augment $\mathcal{S}$ with artificial states to recover the continuous increase property and yet maintain the characteristics of the original solution space. We denote the augmented solution space $\hat{\mathcal{S}}=\mathcal{S} \cup \mathcal{A}$, where $\mathcal{S}$ and $\mathcal{A}$ are mutually exclusive sets respectively consisting of real states and artificial states. The appropriate augmentation scheme involves inserting an appropriate artificial state at each level skipped between pairs of neighboring states.

If $\Theta$ does not possess the continuous increase property, then there exists at least one pair of states, $x \in\left(f_{i}, p_{i}\right)$ and $w \in\left(f_{i+l}, p_{i+l}\right)$, such that $w \in N(x)$ and $l>1$; see Figure 2 . For every such pair, we insert artificial states $(x, i+1, w) \in L\left(f_{i+1}, p_{i+1}\right),(x, i+2, w) \in L\left(f_{i+2}, p_{i+2}\right), \ldots$, and $(x, i+l-1, w) \in$ $L\left(f_{i+l-1}, p_{i+l-1}\right)$ into the solution space. The neighborhood structure is altered so that $(x, i+1, w) \in \hat{N}(x)$ is generated with probability $\hat{\Gamma}(x,(x, i+1, w))=\Gamma(x, w)$ and $\hat{\Gamma}(x, w)=0$. Additionally, $(x, i+2, w) \in$ $\hat{N}(x, i+1, w)$ with $\hat{\Gamma}((x, i+1, w),(x, i+2, w))=1, \ldots$, and $w \in \hat{N}(x, i+l-1, w)$ with $\hat{\Gamma}((x, i+l-1, w), w)=1$.

To keep the augmentation of artificial states from altering the behavior of $(Y(k), T(k))$, we design a Markov chain such that the artificial states have holding times of zero time units while the real states have holding times of one time unit. This is the motivation for the clock time component of our Markov chain. For example, consider the sample path: $Y(k)=x_{0}, Y(k+1)=x_{1}, Y(k+2)=x_{2}, Y(k+3)=x_{3}$. If $x_{0}, x_{1}, x_{2}, x_{3} \in \mathcal{S}$ and $T(k)=t$, then the corresponding sequence of clock times is: $T(k+1)=t+1$, 
$T(k+2)=t+2, T(k+3)=t+3$. However, if $x_{0}, x_{1}, x_{3} \in \mathcal{S}$ and $x_{2} \in \mathcal{A}$ instead, then $T(k+1)=t+1$, $T(k+2)=t+1, T(k+3)=t+2$.

To be precise, the one-step transition probabilities can be defined as follows. For $x \in \mathcal{S}$ and $y \in \hat{\mathcal{S}} \backslash\{x\}$,

$$
P\{Y(k+1)=y, T(k+1)=s \mid Y(k)=x, T(k)=t\}=\begin{aligned}
& \hat{\Gamma}(x, y) \eta\left(\left[v_{t}(y)-v_{t}(x)\right]^{+}\right) I\{s-t=I\{y \in \mathcal{S}\}\},
\end{aligned}
$$

and

$$
P\{Y(k+1)=x, T(k+1)=t+1 \mid Y(k)=x, T(k)=t\}=
$$

$$
1-\sum_{z \neq x} \hat{\Gamma}(x, z) \eta\left(\left(v_{t}(z)-v_{t}(x)\right)^{+}\right)
$$

Now suppose the algorithm is in state $(x, i, w) \in \mathcal{A}$ at step $k$. The algorithm generates a neighbor solution, $y$, with probability $\hat{\Gamma}((x, i, w), y)$ and accepts this state with probability $\eta\left(\left[v_{T(k)}(y)-v_{T(k)}(x, i, w)\right]^{+}\right)$. If the move to $y$ is rejected, then the process descends to $x$. More precisely, for $(x, i, w) \in \mathcal{A}$ and $y \in \hat{\mathcal{S}} \backslash\{x\}$,

$$
\begin{aligned}
& P\{Y(k+1)=y, T(k+1)=s \mid Y(k)=(x, i, w), T(k)=t\}= \\
& \hat{\Gamma}((x, i, w), y) \eta\left(\left[v_{t}(y)-v_{t}(x, i, w)\right]^{+}\right) I\{s-t=I\{y \in \mathcal{S}\}\},
\end{aligned}
$$

and

$$
\begin{aligned}
P\{Y(k+1)=x, T(k+1)=t+1 \mid Y(k)=(x, i, w), T(k)= & t\}= \\
& 1-\sum_{z} \hat{\Gamma}((x, i, w), z) \eta\left(\left[v_{t}(z)-v_{t}(x, i, w)\right]^{+}\right) .
\end{aligned}
$$

In the augmented solution space, $\hat{\mathcal{S}}$, there is a positive probability of the process transitioning from an artificial state $(x, i, w)$ to the state $x \neq(x, i, w)$ at any time $t \geq 0$. That is, $x$ is a "neighbor" of $(x, i, w)$ although $\hat{\Gamma}((x, i, w), x)=0$. To remedy this contradiction, we define neighborhoods for artificial states differently than neighborhoods for real states.

Assumption 3. For any state $z \in \mathcal{A}$, a state $x \in \hat{N}(z) \subset \hat{\mathcal{S}}$ if and only if either

(a) $\hat{\Gamma}(z, x)=1$ ( $x$ is the state generated for consideration), or

(b) $P\{Y(k+1)=x, T(k+1)=t+1 \mid Y(k)=z, T(k)=t\}>0$ ( $x$ is the last real state visited).

The above procedure allows us to recover the continuous-increase property while maintaining the transition probabilities between real states. So we henceforth assume, without loss of generality, that the continuous-increase property holds.

\subsection{Definition of Concepts}

In this section, we use insight provided by the constant ordering of the topography, $\Theta_{\lambda}$, for $\lambda>\lambda^{*}$ to define structural concepts. In particular, we extend the Markov chain concepts of accessibility and communication 
to account for the dynamic solution topography. These definitions extend the framework in Hajek (1988) to allow for infeasible states.

We say that state $y$ is accessible at level $(f, p)$ from state $x$ if (i) $x=y$ and $(f(x), p(x)) \preceq(f, p)$, or (ii) there is a sequence of states $x=x_{0}, x_{1}, \ldots, x_{s}=y$ for some $s \geq 1$ such that $x_{n+1} \in N\left(x_{n}\right)$ for $0 \leq n<s$ and $\left(f\left(x_{n}\right), p\left(x_{n}\right)\right) \preceq(f, p)$ for $0 \leq n \leq s$.

A topology $(\mathcal{S}, f, p, N)$ is level irreducible if $(\mathcal{S}, N)$ is irreducible and, for any level $(f, p)$ and any two states $x, y \in S, x$ is accessible at level $(f, p)$ from $y$ if and only if $y$ is accessible at level $(f, p)$ from $x$. Note that symmetric neighborhoods are sufficient, but not necessary, for level irreducibility.

We say that a state $x$ is a local minimum if there does not exist a state $y$ accessible at level $(f(x), p(x))$ from $x$ such that $(f(y), p(y)) \prec(f(x), p(x))$. For a local minimum $x$, suppose $\left(f_{x}^{\prime}, p_{x}^{\prime}\right) \succ(f(x), p(x))$ is the lowest level at which a state $y$ with $(f(y), p(y)) \prec(f(x), p(x))$ is accessible. Then, the static depth of local minimum $x$ is $S(x)=f_{x}^{\prime}-f(x)$, and the penalty depth of local minimum $x$ is $P(x)=p_{x}^{\prime}-p(x)$. Therefore, each local minimum $x$ is described by a depth pair $(S(x), P(x))$.

We compare the depth pairs of local minima $x$ and $y$ via the $\succ$ operator. If $(S(x), P(x)) \succ(S(y), P(y))$, then we say that the local minimum $x$ is "deeper" than the local minimum $y$. We define the dynamic depth of local minimum $x$ at time $t$ as $d_{t}(x)=S(x)+\lambda_{t} P(x)$. For $t \geq t^{*}, d_{t}(x)$ is interpreted as the smallest distance that the process would have to climb from a local minimum $x$ to reach a local minimum $z$ such that $(f(z), p(z)) \prec(f(x), p(x))$.

For a local minimum $x$, recall $\left(f_{x}^{\prime}, p_{x}^{\prime}\right) \succ(f(x), p(x))$, and thus $(S(x), P(x)) \succ(0,0)$. For a state $y$ that is not a local minimum, there exists a state $z$ with $(f(z), p(z)) \prec(f(y), p(y))$ that is accessible at level $(f(y), p(y))$ from $y$. Thus, any state $y$ that is not a local minimum has a depth pair $(S(y), P(y))=(0,0)$. On the other hand, if a local minimum $x$ is also a global minimum, then $(S(x), P(x))$ is defined to be $(+\infty,+\infty)$.

A cup $C$ is a set of states such that for some level $(f, p)$ and some $x$ such that $(f(x), p(x)) \preceq(f, p)$, $C=\{y: y$ is accessible at level $(f, p)$ from $x\}$. By level irreducibility, one can take the state " $x$ " in the definition of the cup $C$ to be any element of $C$.

To facilitate our analysis of cups, we define some cup-specific quantities. Let the bottom of a cup $C$ be defined as $B=\{x \in C:(f(x), p(x)) \preceq(f(y), p(y)) \forall y \in C\}$. Let the rim of a cup $C$ be defined as $R=\{x \in C:(f(x), p(x)) \succeq(f(y), p(y)) \forall y \in C\}$. That is, $B$ is the set of states in the lowest level of the cup, and $R$ is the set of states in the highest level of the cup. Let the froth of a cup $C$ be $F=\{y$ : $y \notin C$ and $y \in N(x)$ for some $x \in C\}$. Note that $F$ is empty if and only if $C=\mathcal{S}$. We are particularly interested in the states in $R$ that have neighbors in $F$. Formally, let this set of "escape states" be denoted $R^{e}=\{x \in R: N(x) \cap F \neq \emptyset\}$. By the continuous increase property, the process escapes $C$ by jumping from a state in $R^{e}$ to a state in $F$. The sets $B, R$, and $F$ are cup-specific, i.e., they are more accurately denoted $B(C), R(C)$, and $F(C)$. We notationally suppress this dependence, unless discussing more than one cup at a time.

For a cup $C \neq \mathcal{S}$, we define the static depth $S=f(y)-f(x)$ and the penalty depth $P=p(y)-p(x)$, 


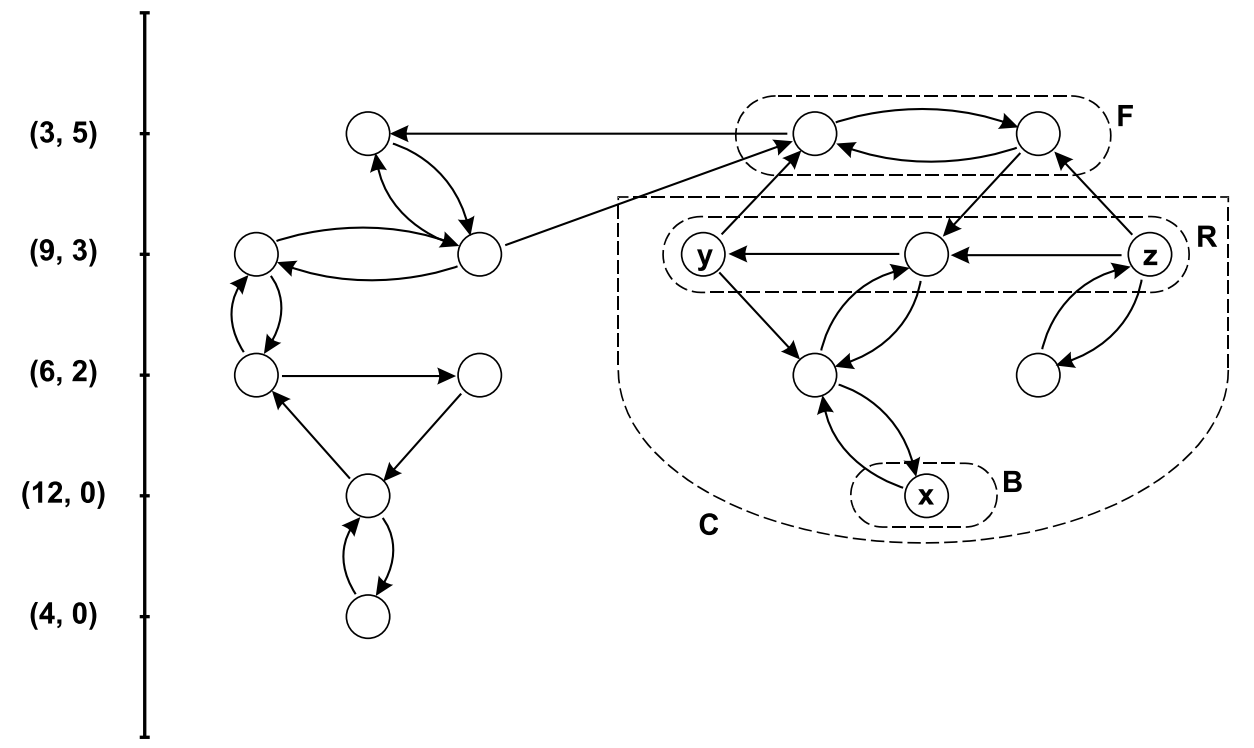

Figure 3: A cup $C$ with depth pair $(S, P)=(S(x), P(x))=(-9,5)=[(3,5)-(12,0)]$. Note that $R^{e}=\{y, z\}$.

where $y \in F$ and $x \in B$. Note that for any cup $C \neq \mathcal{S},(S, P) \succ(0,0)$ implying that either (i) $P>0$ and $S$ is unrestricted, or (ii) $P=0$ and $S>0$. We say that cup $C$ with depth pair $(S, P)$ is deeper than cup $C^{\prime}$ with depth pair $\left(S^{\prime}, P^{\prime}\right)$ if $(S, P) \succ\left(S^{\prime}, P^{\prime}\right)$. Figure 3 illustrates a cup and related concepts.

We define the dynamic depth of cup $C$ at time $t$ as $d_{t}=S+\lambda_{t} P$. For $t \geq t^{*}, d_{t}(C)$ is the distance that the process would have to climb to reach some state $y \in F$ from some state $x \in B$. In accordance with the above definitions, the deepest cup $C$ such that a local minimum $x$ is in the bottom of $C$ has depth pair $(S, P)=(S(x), P(x))$.

Let the "rim height" $r_{t}(C)$ be the distance at time $t$ from a state $u \in R$ to $z \in F$, i.e., $r_{t}=f(z)-f(u)+$ $\lambda_{t}(p(z)-p(u))$. Similarly, let the "girth," $g_{t}(C)$, be the distance at time $t$ from a state $x \in B$ to $u \in R$, i.e., $g_{t}=f(u)-f(x)+\lambda_{t}(p(u)-p(x))$. In this way, the dynamic depth of cup $C$ at time $t$ can be decomposed into two pieces, $d_{t}=r_{t}+g_{t}=S+\lambda_{t} P$.

Note that cups are defined independently of $\lambda_{t}$, although the depths of cups depend on $\lambda_{t}$ and only are intuitive for times $t \geq t^{*}$. The deepening of cup $C$ from time $t$ to time $t+1$, given by $d_{t+1}-d_{t}=P\left(\lambda_{t+1}-\lambda_{t}\right)$, is governed by its penalty depth and the compression schedule.

\subsection{Necessary and Sufficient Condition}

With the model structure and language in place, we are ready to state our main result.

Theorem 1. If the topology $\Theta$ is level irreducible, then the following statements hold.

(a) For any state $x$ that is not a local minimum, $\lim _{k \rightarrow \infty} P\{Y(k)=x\}=0$.

(b) Let $B$ be the bottom of a cup $C$ of static depth $S$ and penalty depth $P$ so that the states in $B$ are local 
minima of static depth $S$ and penalty depth $P$. Then,

$$
\lim _{k \rightarrow \infty} P\{Y(k) \in B\}=0
$$

if and only if

$$
\sum_{k=0}^{\infty} \eta\left(d_{k}^{+}\right)=\sum_{k=0}^{\infty} \eta\left(\left[S+\lambda_{k} P\right]^{+}\right)=+\infty
$$

(c) Let $\left(S^{*}, P^{*}\right)$ be the maximum depth of all states which are local, nonglobal minima. Let $\mathcal{G}^{*}$ denote the set of global minima. Then

$$
\lim _{k \rightarrow \infty} P\left\{Y(k) \in \mathcal{G}^{*}\right\}=1
$$

if and only if

$$
\sum_{k=0}^{\infty} \eta\left(\left[S^{*}+\lambda_{k} P^{*}\right]^{+}\right)=+\infty
$$

Part (a) of Theorem 1 states that the compressed annealing chain is unlikely to reside in a state that is on a "valley wall" in the solution topography. Part (b) declares that the compressed annealing algorithm is unlikely to reside in a local minimum with depth pair less than or equal to $(S, P)$ if and only if the system is cooled and compressed slowly enough. We use the "positive part" operator in the sum of escape probabilities to avoid situations where $S+\lambda_{k} P<0$, which can only occur for small $k$. This operator does not play a role as $k$ tends to infinity. Part (c) is a direct consequence of parts (a) and (b).

\subsubsection{Cooling and Compression Schedules}

Suppose the probability of accepting a nonimproving solution is given by the function $\eta_{k}=\exp \left(-1 / \tau_{k}\right)$. Then the necessary and sufficient condition for convergence is

$$
\sum_{k=0}^{\infty} \exp \left(-\left[S^{*}+\lambda_{k} P^{*}\right]^{+} / \tau_{k}\right)=\infty
$$

Hajek (1988) demonstrates that a cooling schedule of the form $\tau_{k}=c / \ln (k+1)$ for $k \geq 1$ satisfies this condition if $c$ is a constant greater than or equal to the depth of the deepest local, nonglobal minimum. Thus, if we consider a compression schedule such that $\lim _{k \rightarrow \infty} \lambda_{k}=\bar{\lambda} \geq \lambda^{*}$, where $\lambda^{*}$ is defined in Lemma 1 , Hajek's cooling schedule still satisfies Equation (5) if $c \geq S^{*}+\bar{\lambda} P^{*}$.

However, if $P^{*}>0$ and we allow $\lim _{k \rightarrow \infty} \lambda_{k}=\infty$, the depths of local minima also grow to infinity and therefore Hajek's cooling schedule does not satisfy the necessary and sufficient condition. In this case, one particular set of joint cooling and compression schedules that satisfy the necessary and sufficient condition is

$$
\tau_{k}=\frac{\left(S^{*}+\phi\right)+\lambda_{k} P^{*}}{\ln (k+2)}
$$

for $k \geq 0$, where $\lambda_{k}$ grows to infinity slower than $O(\ln k)$ and $\phi \geq 0$ is appropriately defined to ensure that $\left\{\tau_{k}\right\}$ is decreasing. 
While cooling and compression schedules that satisfy the necessary and sufficient condition are often empirically ineffective on practically sized problems, our theoretical structure still contributes to the practice of compressed annealing. Equation (5) suggests compression and cooling schedules with rates of change that decrease over time. In addition, it is clear that the performance of compressed annealing is affected by the dynamic depths of local minima, and in particular, the depth pair of the deepest local, nonglobal minimum, $\left(S^{*}, P^{*}\right)$. This suggests that approximations of depth pairs and the pressure cap $\left(\lambda^{*}\right)$ supply insight on the impact of compression on the solution topography and further guide the practitioner's specification of temperature and pressure. These insights have proven valuable in empirical work on constrained truck fleet replacement and vehicle routing with time windows. See also the motivation for our results given in the introduction.

\subsubsection{Outline of Proof}

The proof of Theorem 1, given in Section 7, follows from three theorems we present in Sections 4, 5, and 6 . In this section, we describe the key ideas behind these three major steps.

In Section 4, we establish Theorem 2 which states that under appropriately slow cooling and compression: (a) the sum of jump probabilities over the number of steps required to escape a cup $C$ is bounded in expectation, and (b) there is a positive uniform lower bound on the probability of exiting the cup via any particular state in the cup's froth. To prove Theorem 2, we begin by partitioning a cup $C$ into its rim and its underlying cups. Structuring a cup in this manner accommodates the use of induction. The proof of part (a) rests on the fact that by the induction hypothesis, $Y$ escapes any underlying cup within cup $C$ by entering a state in the rim of $C$, and on these trips to $R(C), Y$ occasionally hits a state in $R^{e}(C)$. Once $Y$ is in $R^{e}(C)$, the process can escape cup $C$ in a single transition, so if $Y$ visits $R^{e}(C)$ often enough, the process will eventually escape $C$, and escape quickly enough to ensure the expectation is bounded.

To show part (b) of Theorem 2, we consider some state $\bar{x} \in R^{e}(C)$ with $\bar{y} \in N(\bar{x}) \cap F$ and recognize that if $P\{Y$ escapes $C$ through $\bar{x}\}>\delta>0$, then $P\{Y$ enters $\bar{y}$ upon exiting $C\}$ is also bounded away from zero. We prove that the $P\{Y$ escapes $C$ via $\bar{x}\}$ has a positive lower bound by showing that, for some constant $c$,

$P\{Y$ escapes $C$ via $\bar{x}$ after hitting $\bar{x} i$ times $\} \geq$

$c P\left\{Y\right.$ escapes $C$ from another state in $R^{e}$ after hitting $\bar{x} i$ times $\}$,

and then summing over $i$. Since every state $y \in F$ is a neighbor of some $x \in R^{e}$, part (b) follows directly.

Section 5 develops Theorem 3 which presents a lower bound on the probability of remaining in a cup over a given number of iterations. To achieve this result, we partition a cup $C$ into a set $D$ and a collection of cups $\left\{C_{1}, \ldots, C_{n}\right\}$. The set $D$ is defined such that for $x \in D$, the bottom of $C$ is accessible at level $(f(x), p(x))$. Alternatively, for $x \in C_{i},(f, p) \succ(f(x), p(x))$ is the lowest level such that the bottom of $C$ is accessible at $(f, p)$ from $x$. Viewing the structure of $C$ in this way, we see that $Y$ can escape the cup or hit the bottom only through states in $D$. We proceed by utilizing a time-homogeneous birth-and-death process 
(that stochastically dominates $(Y, T)$ ) to determine an upper bound on the probability that $Y$ escapes $C$ before hitting the bottom. This upper bound on $P\{Y$ escapes $C$ before hitting $B\}$ is then used to develop the lower bound on the probability of remaining in the cup over $n$ iterations as stated in Theorem 3 .

In Section 6, Theorem 4 provides the capstone to the proof of Theorem 1. Theorem 4 establishes $\sum_{k=0}^{\infty} \eta\left(S+\lambda_{k} P\right)=+\infty$ as the sufficient condition for convergence in probability to the set of states composing the lower portions of cups deeper than $(S, P)$. Define $E_{S, P}$ as the set of local minima deeper than $(S, P)$. To establish this sufficient condition, we partition the solution space $\mathcal{S}$ into a set $J$ and a collection of cups $\left\{C_{1}, \ldots, C_{l}\right\}$. The set $J$ is defined such that for any $x \in J$, there exists a state in $E_{S, P}$ that is accessible at level $(f(x), p(x))$. Alternatively, for $x \in C_{j},(f, p) \succ(f(x), p(x))$ is the lowest level such that a state in $E_{S, P}$ is accessible at $(f, p)$ from $x$. Furthermore, the depth of any cup in the collection $\left\{C_{1}, \ldots, C_{l}\right\}$ is at most $(S, P)$.

Due to the partitioning of $\mathcal{S}$, the process hits $J$ upon exiting any cup $C_{j}$, and from any state $x \in J$, there exists a path to $E_{S, P}$ that rises no higher than $(f(x), p(x))$. Thus, we use Theorem 2 to show that $Y$ escapes any cup $C_{j}$, enters a state in $J$, and eventually reaches a local minimum deeper than $(S, P)$. In particular, we give a lower bound on the probability of reaching $E_{S, P}$ within a given number of iterations. Applying Theorem 3, we get a lower bound on the probability of remaining "near" $E_{S, P}$ over a given number of iterations. Combining these two observations in a limiting interval argument, we obtain the result of Theorem 4.

\section{Climbing the Increasing Depths}

In this section, we analyze the ability of the process to escape deepening cups. For a given $\operatorname{cup} C$, define the step at which the process first escapes $C$ as $W=\inf \{k \geq 0: Y(k) \in F\}$.

Theorem 2. There exist $\epsilon>0$ and $0<\xi \leq 1$ depending only on $\Theta$ and $C$ so that for every time $t_{0} \geq t^{*}$, every $x_{0} \in C$ where $C$ has depth pair $(S, P)$, every $y_{0} \in F$, and every $\left(\eta_{t}, \lambda_{t}: t \geq 0\right)$ such that $\eta\left(r_{t_{0}}\right) \leq \xi$ and $\sum_{k=0}^{\infty} \eta\left(d_{t_{0}+k}\right)=+\infty$,

$$
\begin{gathered}
\text { (a) } E\left[\sum_{k=0}^{W} \eta\left(d_{T(k)}\right) \mid(Y(0), T(0))=\left(x_{0}, t_{0}\right)\right] \leq \frac{1}{\epsilon} \text {, and } \\
\text { (b) } P\left\{Y(W)=y_{0} \mid(Y(0), T(0))=\left(x_{0}, t_{0}\right)\right\} \geq \epsilon
\end{gathered}
$$

In Hajek (1988), an analogous result is proved for the situation where pressure is constant over time. We generalize this result to handle deepening cups.

As in Theorem 3 of Hajek (1988), Theorem 2 is proved by strong induction via a sequence of lemmas. As shown in Figure 4, the states in a cup $C$ can be partitioned into its rim, $R$, and a collection of underlying cups, $C_{1}, C_{2}, \ldots, C_{n}$. Any of the cups nested within $C$ have a depth pair $\left(S\left(C_{i}\right), P\left(C_{i}\right)\right) \prec(S(C), P(C))$. 


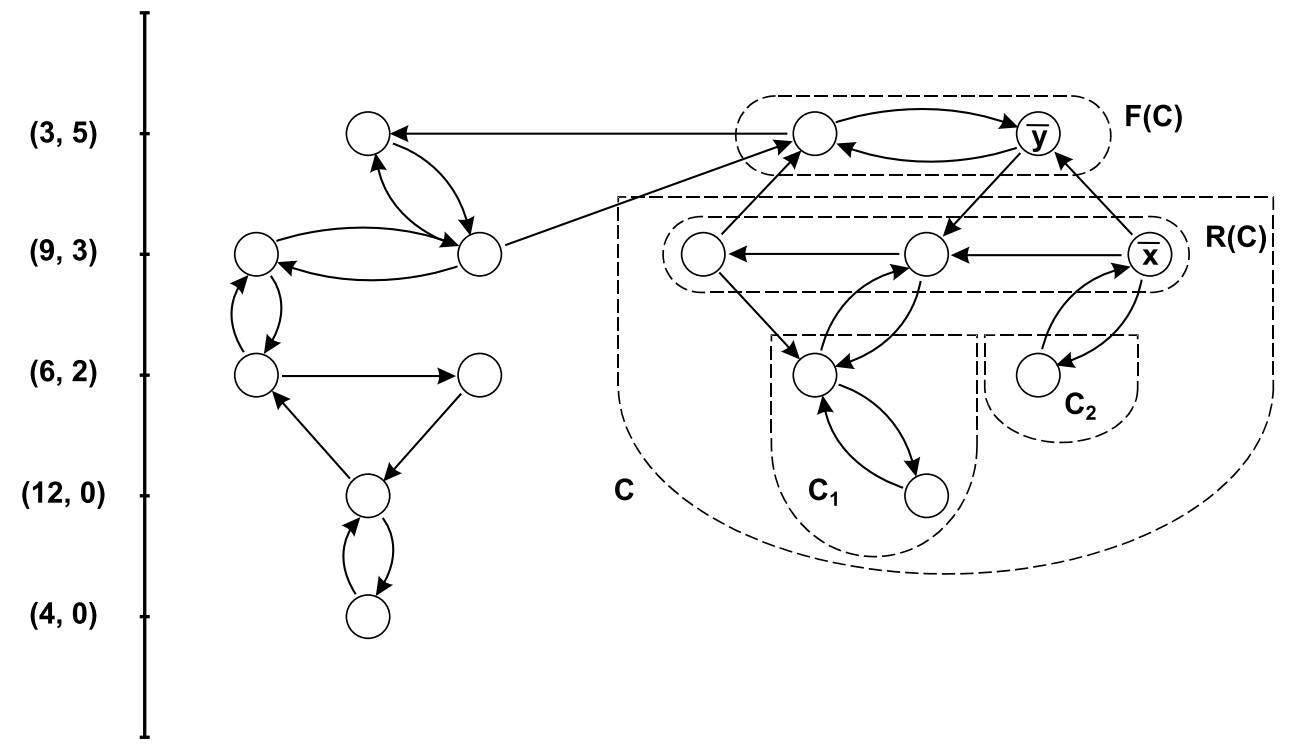

Figure 4: Cup $C$ is partitioned into its $\operatorname{rim}(R(C))$ and a collection of shallower cups $\left(C_{1}\right.$ and $\left.C_{2}\right)$.

Furthermore, by the continuous increase property, when the process first escapes a nested $\operatorname{cup} C_{i}$, it must visit a state in $R$.

Induction Hypothesis: Theorem 2 is true for any cup $C^{\prime}$ with $\left(S\left(C^{\prime}\right), P\left(C^{\prime}\right)\right) \prec(S(C), P(C))$.

Base Case: Cup $C$ consists only of states at a single level, i.e., $C=R$.

The proof of Theorem 2 for the base case is similar to that of the induction step. We continue with the exposition of the general case and omit the details of the base case. We need some preliminaries before proceeding to the proof of Theorem 2 .

To track the process when it enters the $\operatorname{rim}$ of $C$, we define $\left\{J_{i}\right\}$ such that $J_{0}=0$ and $J_{i+1}=\inf \{k>$ $\left.J_{i}: Y(k) \in R\right\} \wedge W$ for $i \geq 0$. Observe that $J_{i}$ is the iteration at which the process visits $R$ for the $i^{\text {th }}$ time or finally escapes the cup.

Since the dynamic depth of any cup $C_{i}$ at time $t \geq t^{*}$ is no deeper than $g_{t}(C)$ which is less than $d_{t}(C)$, we can apply the induction hypothesis. Therefore, we get $\epsilon_{i}$ and $\xi_{i}$ for each cup $C_{i}$ such that if $\eta\left(r_{t_{0}}\left(C_{i}\right)\right) \leq \xi_{i}$ and $x_{0} \in C_{i}$, then

$$
E\left[\sum_{k=0}^{J_{1}} \eta\left(g_{T(k)}\right)\right] \leq \frac{1}{\epsilon_{i}} .
$$

(For notational convenience, we define all probabilities and expectations with respect to the probability measure induced when $(Y(0), T(0))=\left(x_{0}, t_{0}\right)$ as defined in the statement of Theorem 2.)

Furthermore, for every $y$ such that $y \in R \cap N(x)$ for $x \in C_{i}$,

$$
P\left\{Y\left(J_{1}\right)=y\right\} \geq \epsilon_{i} .
$$

Therefore, we can set $\hat{\epsilon}=\min _{i} \epsilon_{i}$ and $\hat{\xi}=\min _{i} \xi_{i}$, so that if $\eta\left(r_{t_{0}}\left(C_{i}\right)\right) \leq \hat{\xi}$ and $x_{0} \in C_{i}$ for some $i=1, \ldots, n$, 
then

$$
E\left[\sum_{k=0}^{J_{1}} \eta\left(g_{T(k)}\right)\right] \leq \frac{1}{\hat{\epsilon}}
$$

and for every $y$ such that $y \in R \cap N(x)$ for $x \in C_{i}$,

$$
P\left\{Y\left(J_{1}\right)=y\right\} \geq \hat{\epsilon}
$$

Let $K_{0}=0$ and define $K_{i+1}=\left(\inf \left\{k>K_{i}: Y(k) \in R^{e}\right\} \wedge W\right)$ for $i \geq 0$. Iteration $K_{i}$ is the step at which the process visits $R^{e}$ for the $i^{\text {th }}$ time or finally escapes the cup.

We describe the history of the process up to step $J_{k}$ through the $\sigma$-algebra $\mathcal{F}_{k}$ generated by $\left\{\left(Y(i): 0 \leq i \leq J_{k}\right), T(0)\right\}$. Note that

$$
\begin{aligned}
\mathcal{F}_{k} & =\sigma\left\{\left(Y(i): 0 \leq i \leq J_{k}\right), T(0)\right\} \\
& =\sigma\left\{(Y(i), T(i)): 0 \leq i \leq J_{k}\right\} .
\end{aligned}
$$

That is, given the sequence of states and the initial clock time, we can compute the clock time at each step in the entire sequence.

Lemma 2 states that once in a cup, the expected number of visits to states in $R$ until reaching a state in $R^{e}$ is bounded above by a constant. Let $M=\inf \left\{j>0: Y\left(J_{j}\right) \in R^{e} \cup F\right\}$. The definition of $M$ includes the set $F$ to account for the case in which $Y(0)=x_{0} \in R^{e}$ and $Y(1) \in F$.

Lemma 2. Under the conditions of Theorem 2, for any $x_{0} \in C, E[M] \leq c_{1}$ for some constant $c_{1}$ depending only on $\Theta$ and $C$.

Proof. From the induction hypothesis of Theorem 2, we know that if the process is in any state $x \in C_{i}$ at any time $t \geq t^{*}$, then with probability one it will eventually hit a state in $R$. It also follows from the induction hypothesis that there exists $\bar{\epsilon}>0$ depending only on $\Theta$ and $C$ so that, for every $x, y \in R$ that can communicate without visiting another state in $R, P\left\{Y\left(J_{k+1}\right)=y \mid \mathcal{F}_{k}\right\} \geq \bar{\epsilon}$ on the event $\left\{Y\left(J_{k}\right)=x\right\}$. A geometric trials argument incorporating these two observations supplies the desired result.

Lemma 3 essentially states that the expected jump probabilities accumulated between entrances into the cup's rim until hitting an "escape state" is bounded above by a constant.

Lemma 3. Under the conditions of Theorem 2, there exists a constant $c_{2}$ depending only on $\Theta$ and $C$ such that

$$
E\left[\sum_{s=J_{k}+1}^{J_{k+1}} \eta\left(g_{T(s)}\right) I\left\{s \leq K_{1}\right\} \mid \mathcal{F}_{k}\right] \leq c_{2} .
$$

Proof. Note that $Y\left(J_{k}+1\right)$ is the state to which the system transitions immediately after visiting $R$ for the $k$ th time, while $Y\left(J_{k+1}\right)$ is the state of the process when in $R \cup F$ for the $(k+1)$ st time. Considering the two scenarios, $Y\left(J_{k}+1\right) \notin R \cup F$ or $Y\left(J_{k}+1\right)=Y\left(J_{k+1}\right) \in R \cup F$, the result follows from the induction hypothesis. 
Lemma 4 states that the expected jump probabilities accumulated until reaching a state in the cup's rim that has a neighbor outside the cup is bounded by a constant times the probability of accepting a jump from the rim of the cup to the froth at time $t_{0}$.

Lemma 4. Under the conditions of Theorem 2, there is a constant $c_{3}$ depending only on $\Theta$ and $C$ such that for every $x_{0} \in C$, and every $t_{0} \geq t^{*}$ with $\eta\left(r_{t_{0}}\right) \leq \xi$,

$$
E\left[\sum_{s=0}^{K_{1}} \eta\left(d_{T(s)}\right)\right] \leq c_{3} \eta\left(r_{t_{0}}\right) .
$$

Proof. Consider the stochastic process $\left(Z_{k}: k \geq 0\right)$, where $Z_{0}=0$ and for $k>0$

$$
Z_{k}=M \wedge k-\frac{1}{c_{2}} \sum_{s=0}^{J_{k}} \eta\left(g_{T(s)}\right) I\left\{s \leq K_{1}\right\} .
$$

With an application of Lemma 3 for the case when $k<M$, and the fact that $K_{1}=J_{M}$ for $k \geq M$, it is straightforward to show that $\left(Z_{k}: k \geq 0\right)$ is a submartingale with respect to $\left(\mathcal{F}_{k}: k \geq 0\right)$. Therefore,

$$
E\left[\frac{1}{c_{2}} \sum_{s=0}^{J_{k}} \eta\left(g_{T(s)}\right) I\left\{s \leq K_{1}\right\}\right] \leq E[M \wedge k] \leq E[M] \forall k .
$$

By the monotone convergence theorem and Lemma 2,

$$
E\left[\sum_{s=0}^{K_{1}} \eta\left(g_{T(s)}\right)\right] \leq c_{2} c_{1} \equiv c_{3} .
$$

Now observe that since $\eta\left(r_{t}\right)$ is decreasing for all $t \geq t^{*}$, and $T(0)=t_{0} \geq t^{*}$,

$$
\frac{\eta\left(d_{T(s)}\right)}{\eta\left(r_{t_{0}}\right)}=\frac{\eta\left(r_{T(s)}\right) \eta\left(g_{T(s)}\right)}{\eta\left(r_{t_{0}}\right)} \leq \eta\left(g_{T(s)}\right)
$$

The result now follows from (6).

In Lemma 5, we show that the expected jump probabilities accumulated over several visits to the set $R^{e}$ is bounded above by a constant.

Lemma 5. Under the conditions of Theorem 2, for $1 \leq i \leq j<+\infty$

$$
E\left[\sum_{s=K_{i}+1}^{K_{j}} \eta\left(d_{T(s)}\right) \mid Y\left(K_{i-1}\right), T\left(K_{i-1}\right)\right] \leq \frac{c_{3}}{c_{0}} .
$$

Proof. The proof is by reverse induction. The result is trivial if $i=j$. So suppose it is true for $i+1$ with $1 \leq i+1 \leq j$. Define $\Phi(i, j)=\sum_{s=K_{i}+1}^{K_{j}} \eta\left(d_{T(s)}\right)$. From Lemma 4 we have that

$$
E\left[\Phi(i, i+1) \mid Y\left(K_{i}\right), T\left(K_{i}\right)\right] \leq c_{3} \eta\left(r_{T\left(K_{i}\right)}\right)
$$

on the event that $Y\left(K_{i}\right) \in C$ and is 0 otherwise. The inductive hypothesis gives that

$$
E\left[\Phi(i+1, j) \mid Y\left(K_{i}\right), T\left(K_{i}\right)\right] \leq \frac{c_{3}}{c_{0}}
$$


on the event $Y\left(K_{i}\right) \in C$ and is 0 otherwise. Thus

$$
\begin{aligned}
E & {\left[\Phi(i, j) \mid Y\left(K_{i-1}\right), T\left(K_{i-1}\right)\right] } \\
& =E\left[E\left[\Phi(i, i+1)+\Phi(i+1, j) \mid Y\left(K_{i}\right), T\left(K_{i}\right), Y\left(K_{i-1}\right), T\left(K_{i-1}\right)\right] \mid Y\left(K_{i-1}\right), T\left(K_{i-1}\right)\right] \\
& \leq E\left[\left(c_{3} \eta\left(r_{T\left(K_{i}\right)}\right)+\frac{c_{3}}{c_{0}}\right) I\left(Y\left(K_{i}\right) \in C\right) \mid Y\left(K_{i-1}\right), T\left(K_{i-1}\right)\right] \\
& \leq E\left[\left(c_{3} \eta\left(r_{T\left(K_{i-1}\right)}\right)+\frac{c_{3}}{c_{0}}\right) I\left(Y\left(K_{i}\right) \in C\right) \mid Y\left(K_{i-1}\right), T\left(K_{i-1}\right)\right] \\
& \left.\leq \frac{c_{3}}{c_{0}} P\left\{Y\left(K_{i}\right) \in C \mid Y\left(K_{i-1}\right), T\left(K_{i-1}\right)\right\}+c_{3} \eta\left(r_{T\left(K_{i-1}\right)}\right)\right) \\
& \left.\leq \frac{c_{3}}{c_{0}}\left(1-c_{0} \eta\left(r_{T\left(K_{i-1}\right)}\right)\right)+c_{3} \eta\left(r_{T\left(K_{i-1}\right)}\right)\right) .
\end{aligned}
$$

The final inequality follows since $P\left(Y\left(K_{i}\right) \notin C \mid Y\left(K_{i-1}\right), T\left(K_{i-1}\right)\right)$ is the probability of directly jumping out of the cup $C$ from $Y\left(K_{i-1}\right)$, which is lower bounded by $c_{0} r_{T\left(K_{i-1}\right)}$.

Proof of Theorem 2(a). Setting $i=1$ in Lemma 5 and letting $j$ tend to infinity, we apply the monotone convergence theorem to get

$$
E\left[\sum_{s=K_{1}+1}^{W} \eta\left(d_{T(s)}\right)\right] \leq \frac{c_{3}}{c_{0}} .
$$

Together with Lemma 4, we get

$$
\begin{aligned}
E\left[\sum_{s=0}^{W} \eta\left(d_{T(s)}\right)\right] & =E\left[\sum_{s=0}^{K_{1}} \eta\left(d_{T(s)}\right)\right]+E\left[\sum_{s=K_{1}+1}^{W} \eta\left(d_{T(s)}\right)\right] \\
& \leq c_{3} \eta\left(r_{t_{0}}\right)+\frac{c_{3}}{c_{0}} \\
& \leq c_{3}+\frac{c_{3}}{c_{0}} .
\end{aligned}
$$

Now we proceed to the proof of Theorem 2(b). Let $\bar{y} \in F$ be fixed. Choose a state $\bar{x} \in R^{e} \subset R$ with $\bar{y} \in N(\bar{x}) \cap F$. Define $L^{*}=\inf \left\{l \geq 0: Y\left(J_{l}\right) \in F\right\}$. We track the process entrances into $\bar{x}$ by letting $L_{0}=0$ and $L_{i+1}=\inf \left\{l>L_{i}: Y\left(J_{l}\right)=\bar{x}\right\} \wedge L^{*}$ for $i \geq 0$. Note that $J_{L_{i}}$ is the iteration at which the process enters $\bar{x}$ for the $i^{\text {th }}$ time or, if $L_{i}=L^{*}$, the iteration at which the process hits a state in $F$. Observe that the events $\left\{J_{L_{i}}=W\right\}$ and $\left\{L_{i}=L^{*}\right\}$ are identical, implying that $Y(W)=Y\left(J_{L^{*}}\right)$.

Lemma 6 shows that the number of times $Y$ visits states in $R \backslash\{\bar{x}\}$ between the $i^{\text {th }}$ and $i+1^{\text {st }}$ visit to $\{\bar{x}\} \cup F$ is bounded above by a constant. The proof is similar to that of Lemma 2 and omitted.

Lemma 6. Under the conditions of Theorem 2, for any $x_{0} \in C$,

$$
E\left[\left(L_{i+1}-L_{i}-1\right)^{+} \mid \mathcal{F}_{L_{i}}\right] \leq c_{4}
$$

for some constant $c_{4}$ depending only on $\Theta$ and $C$.

Define the events $\left(H_{i}: i \geq 0\right)$ by $H_{0}=\left\{J_{L_{1}}=W\right\}$ and for $i \geq 1, H_{i}=\left\{J_{L_{i}}+1<W=J_{L_{i+1}}\right\}$. The event $H_{0}$ occurs if the process escapes the cup without ever visiting $\bar{x}$. The event $H_{i}$ occurs when the process 
visits $\bar{x} i$ times before exiting the cup, but escapes the cup through another state in $R^{e}$. Define the events $\left(G_{i}: i \geq 0\right)$ by $G_{i}=\left\{J_{L_{i}}+1=W\right\}$. The event $G_{0}$ occurs if $Y(0)=x_{0}=\bar{x}$ and $Y(1) \in F$. For $i \geq 1$, the event $G_{i}$ occurs when the process escapes the cup immediately after visiting $\bar{x}$ for the $i^{\text {th }}$ time. In Lemma 7 below, we give bounds on the probabilities of these events.

Lemma 7. Under the conditions of Theorem 2, the following inequalities hold for $c_{4}$ as defined in Lemma 6 and some constant $c_{5}>0$ depending only on $\Theta$ and $C$ :

$$
P\left\{H_{0}\right\} \leq c_{4} \eta\left(r_{t_{0}}\right)
$$

and

$$
P\left\{H_{i}\right\} \leq c_{5} P\left\{G_{i}\right\}
$$

for $i \geq 1$.

Proof. Consider the stochastic process $\left(\Upsilon_{k}: k \geq 0\right)$, where $\Upsilon_{0}=0$ and for $k \geq 1$,

$$
\Upsilon_{k}=I\left\{Y\left(J_{k}\right) \in F\right\}-\sum_{j=0}^{\left(k \wedge L^{*}\right)-1} \eta\left(r_{T\left(J_{j}\right)}\right) .
$$

Observing that $P\left\{Y\left(J_{k+1}\right) \in F \mid \mathcal{F}_{k}\right\} \leq \eta\left(r_{T\left(J_{k}\right)}\right)$ on the event $\left\{k<L^{*}\right\}$ and $Y\left(J_{k}\right) \in F$ for all $k \geq L^{*}$, it is straightforward to show that $\left(\Upsilon_{k}, k \geq 0\right)$ is a supermartingale with respect to $\mathcal{F}_{k}$.

Note that since $I\left\{Y\left(J_{k}\right) \in F\right\}=I\left\{J_{k}=W\right\}$, the event $H_{i}$ occurs if and only if $I\left\{Y\left(J_{L_{i+1}}\right) \in F\right\}-$ $I\left\{Y\left(J_{L_{i}+1}\right) \in F\right\}=1-0=1$. Therefore, we use the optional sampling theorem for supermartingales and the observation that $\eta\left(r_{k}\right)$ is decreasing in $k$ to get

$$
\begin{aligned}
P\left\{H_{i} \mid \mathcal{F}_{L_{i}}\right\} & =E\left[I\left\{Y\left(J_{L_{i+1}}\right) \in F\right\}-I\left\{Y\left(J_{L_{i}+1}\right) \in F\right\} \mid \mathcal{F}_{L_{i}}\right] \\
& \leq E\left[\sum_{j=\left(\left(L_{i}+1\right) \wedge L^{*}\right)}^{\left(L_{i+1} \wedge L^{*}\right)-1} \eta\left(r_{T\left(J_{j}\right)}\right) \mid \mathcal{F}_{L_{i}}\right] \\
& \leq c_{4} \eta\left(r_{T\left(J_{L_{i}}\right)}\right)
\end{aligned}
$$

where $c_{4}$ is defined in Lemma 6 . For $i=0$, this implies (7). For $i \geq 1$, note that

$$
\begin{aligned}
P\left\{G_{i} \mid \mathcal{F}_{L_{i}}\right\} & =I\left\{Y\left(J_{L_{i}}\right)=\bar{x}\right\} \sum_{z \in N(\bar{x}) \cap F} \Gamma(\bar{x}, z) \eta\left(r_{T\left(J_{L_{i}}\right)}\right) \\
& \geq c_{0} \eta\left(r_{T\left(J_{L_{i}}\right)}\right) I\left\{Y\left(J_{L_{i}}\right)=\bar{x}\right\} .
\end{aligned}
$$

Combining the bounds on $G_{i}$ and $H_{i}$ gives (8), where $c_{5}=\frac{c_{4}}{c_{0}}$.

Proof of Theorem 2(b). Observe that if $Y\left(J_{L^{*}-1}\right)=\bar{x}$, then the process escapes the cup $C$ by jumping from $\bar{x} \in R^{e}$. For the event $\left\{Y\left(J_{L^{*}-1}\right)=\bar{x}\right\}$ to occur, $\left\{J_{L_{i}}+1=W\right\}$ for some $i$. Therefore, by Lemma 7 , 
we get

$$
\begin{aligned}
P\left\{Y\left(J_{L^{*}-1}\right)=\bar{x}\right\} & =\sum_{i=0}^{\infty} P\left\{G_{i}\right\} \\
& \geq \frac{1}{c_{5}} \sum_{i=1}^{\infty} P\left\{H_{i}\right\} \\
& \geq \frac{1}{c_{5}}\left(1-P\left\{H_{0}\right\}-P\left\{Y\left(J_{L^{*}-1}\right)=\bar{x}\right\}\right) \\
& \geq \frac{1}{c_{5}}\left(1-c_{4} \eta\left(r_{t_{0}}\right)-P\left\{Y\left(J_{L^{*}-1}\right)=\bar{x}\right\}\right) .
\end{aligned}
$$

Combining like terms, we obtain

$$
P\left\{Y\left(J_{L^{*}-1}\right)=\bar{x}\right\} \geq \frac{1-c_{4} \eta\left(r_{t_{0}}\right)}{1+c_{5}} .
$$

The probability of hitting $\bar{y}$ given we exit the cup via $\bar{x}$ is given by

$$
\begin{aligned}
P\left\{Y(W)=\bar{y} \mid Y\left(J_{L^{*}-1}\right)=\bar{x}\right\} & =\frac{\Gamma(\bar{x}, \bar{y})}{\sum_{w \in N(\bar{x}) \cap F} \Gamma(\bar{x}, w)} \\
& \geq c_{0} .
\end{aligned}
$$

We conclude that

$$
\begin{aligned}
P\{Y(W)=\bar{y}\} & \geq P\left\{Y(W)=\bar{y}, Y\left(J_{L^{*}-1}\right)=\bar{x}\right\} \\
& \geq \frac{c_{0}\left(1-c_{4} \eta\left(r_{t_{0}}\right)\right)}{\left(1+c_{5}\right)}
\end{aligned}
$$

Setting

$$
\epsilon=\min \left\{\frac{c_{0}}{c_{3}\left(1+c_{0}\right)}, \frac{c_{0}\left(1-c_{4} \eta\left(r_{t_{0}}\right)\right)}{\left(1+c_{5}\right)}\right\}
$$

with the caveat that $\eta\left(r_{t_{0}}\right) \leq \xi \leq \frac{1}{c_{4}}$, we complete the proof of Theorem 2 by the principle of induction.

\section{Sinking to the Bottom}

In this section, we analyze the behavior of the process once it has reached a local minimum. In particular, we are interested in the probability of the process remaining in a cup $C$ with $\operatorname{depth}$ pair $(S, P)$ for $q$ iterations. The arguments are based on an analogous result in Hajek (1988) for the continuous-time case with static cup depths.

Theorem 3. There exist $0<\bar{\xi} \leq 1$ and $c_{6}>0$ depending only on $\Theta$ such that for any cup $C$ with bottom $B$, any $x_{0} \in B$ and any $t_{0} \geq t^{*}$ with $\max \left\{\eta_{t_{0}}, \eta\left(d_{t_{0}}\right)\right\} \leq \bar{\xi}$,

$$
P\left\{Y(k) \in C \text { for } 0 \leq k \leq q \mid(Y(0), T(0))=\left(x_{0}, t_{0}\right)\right\} \geq \exp \left(-c_{6} \sum_{k=0}^{q-1} \eta\left(d_{T(k)}\right)\right)
$$




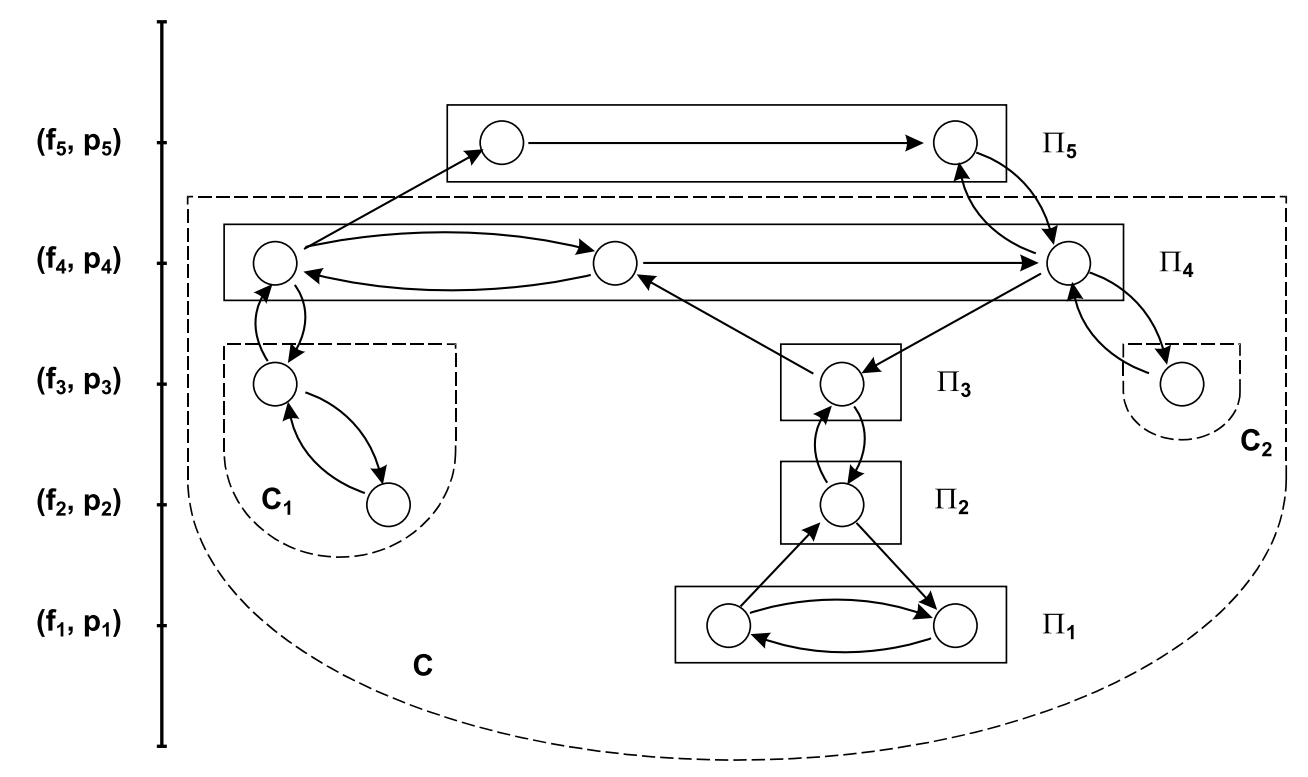

Figure 5: Cup $C$ is partitioned into $D=\cup_{i=1}^{4} \Pi_{i}$ and a collection of smaller cups $\left(C_{1}\right.$ and $\left.C_{2}\right)$.

Since there are only a finite number of cups in $\mathcal{S}$, it suffices to prove Theorem 3 for an arbitrary cup $C$. Recall that the highest level in a cup $C$ is $(f(y), p(y))$, for any $y \in R$, the rim of the cup. For such $y$, if $(f(y), p(y))=\left(f_{\ell}, p_{\ell}\right)$, then the cup $C$ is the entire state space $\mathcal{S}$. Theorem 3 is then trivial.

So, assume that the highest level in $C$ is $\left(f_{r}, p_{r}\right) \prec\left(f_{\ell}, p_{\ell}\right)$. For notational ease we further assume, without loss of generality, that $(f(x), p(x))=\left(f_{1}, p_{1}\right)$. The dynamic depth of the cup $C$ at time $t \geq t^{*}$ is then given by $d_{t}=\left(f_{r}-f_{1}\right)+\lambda_{t}\left(p_{r}-p_{1}\right)$.

Given a state $x \in C$, let $(G(x), Q(x))$ be the lowest level at which a state in $B$ is accessible from $x$. As shown in Figure 5, we can partition the cup $C$ into the set $D$ and cups $C_{1}, \ldots, C_{m}$, where $D=$ $\{x \in C:(f(x), p(x))=(G(x), Q(x))\}$. That is, the set $D$ contains the states in $C$ that can reach the bottom of the cup without "climbing" to a higher level. In fact, if $x \in C$ and $(f(x), p(x)) \prec(G(x), Q(x))$, then $x \in C_{i}$ for some $i$, and $C_{i}$ is the set of all states that are accessible from $x$ at a level strictly lower than $(G(x), Q(x))$.

Define $\Pi_{r+1}=F$, and $\Pi_{i}=\left\{x \in D:(f(x), p(x))=\left(f_{i}, p_{i}\right)\right\}$ for $i=1, \ldots, r$. The set $\Pi_{i}$ exclusively includes states in the set $D$ at level $\left(f_{i}, p_{i}\right)$, i.e., $\Pi_{i}=D \cap L\left(f_{i}, p_{i}\right)$ for $i \leq r$. Furthermore, let the distance between adjacent levels $\left(f_{j+1}, p_{j+1}\right)$ and $\left(f_{j}, p_{j}\right)$ at iteration $k$ be denoted by $\Delta_{j}(T(k))=\left(f_{j+1}-f_{j}+\right.$ $\left.\lambda_{T(k)}\left(p_{j+1}-p_{j}\right)\right)$ for $1 \leq j \leq r$.

Define $\delta=\min \left\{m: Y(m) \in \Pi_{1} \cup \cdots \cup \Pi_{j-1} \cup \Pi_{j+1}\right\}$. Given $Y(0)=x \in \Pi_{j}, \delta$ denotes the first iteration that the process changes levels within $D$. Denote the number of iterations that the process spends in $\Pi_{j}$ before changing levels in $D$ as $\vartheta=\mid\left\{n: 0 \leq n \leq \delta\right.$ and $\left.Y(n) \in \Pi_{j}\right\} \mid$.

Lemma 8. For $2 \leq j \leq r$, let $\bar{x} \in \Pi_{j}$. Under the conditions of Theorem 3, for any $\bar{t} \geq t^{*}, E[\vartheta \mid(Y(0), T(0))=(\bar{x}, \bar{t})] \leq$ 
$c_{7}$ for some constant $c_{7}$ depending only on $\Theta$ and $C$.

Proof. The partitioning of the cup $C$ into sets $\left\{D, C_{1}, \ldots, C_{m}\right\}$ instills the property that $Y$ visits a state in $D$ upon escaping a cup $C_{i}$. Note that $\vartheta$ is not incremented during iterations spent in $C_{i}$. The result follows by a geometric trials argument similar to the proof of Lemma 2 .

Lemma 9. For $2 \leq j \leq r$, let $\bar{x} \in \Pi_{j}$. Under the conditions of Theorem 3, for any $\bar{t} \geq t^{*}$,

$$
P\left\{\delta<\infty, Y(\delta) \in \Pi_{j+1} \mid(Y(0), T(0))=(\bar{x}, \bar{t})\right\} \leq c_{7} \eta\left(\Delta_{j}(\bar{t})\right),
$$

where $c_{7}$ is defined in Lemma 8.

Proof. Consider the stochastic process $\left(\Psi_{k}: k \geq 0\right)$, where $\Psi_{0}=0$ and for $k \geq 1$,

$$
\Psi_{k}=I\left\{Y(k \wedge \delta) \in \Pi_{j+1}\right\}-\sum_{s=0}^{(k \wedge \delta)-1} \eta\left(\Delta_{j}(T(s))\right) I\left\{Y(s) \in \Pi_{j}\right\} .
$$

Note that $P\left\{Y(k+1) \in \Pi_{j+1} \mid \mathcal{O}_{k}\right\} \leq \eta\left(\Delta_{j}(T(k))\right) I\left\{Y(k) \in \Pi_{j}\right\}$ on the event $\{k<\delta\}$, where $\mathcal{O}_{k}=\sigma(Y(i)$ : $0 \leq i \leq k, T(0))$. Using this observation, it is straightforward to show that $\left(\Psi_{k}: k \geq 0\right)$ is a supermartingale with respect to the filtration $\left\{\mathcal{O}_{k}: k \geq 0\right\}$. Using Lemma 8 and the dominated convergence theorem, we get

$$
\begin{aligned}
P & \left\{\delta<\infty, Y(\delta) \in \Pi_{j+1} \mid(Y(0), T(0))=(\bar{x}, \bar{t})\right\} \\
& \leq E\left[\sum_{s=0}^{\delta-1} \eta\left(\Delta_{j}(T(s))\right) I\left\{Y(s) \in \Pi_{j}\right\} \mid(Y(0), T(0))=(\bar{x}, \bar{t})\right] \\
& \leq c_{7} \eta\left(\Delta_{j}(\bar{t})\right) .
\end{aligned}
$$

We stochastically bound the level process in our setting with a time-homogeneous, discrete-time Markov chain. A bound on a certain probability will then follow. We first give the discrete-time Markov chain result, which is essentially identical to Lemma 4.2 in Hajek (1988), and so the proof is omitted.

For cup $C$, define $\Delta(t)=\min \left\{\Delta_{i}(t): 0 \leq i \leq r\right\}$ for some fixed clock time $t \geq t^{*}$. Choose $\bar{\xi}$ with $0<\bar{\xi}<1$ so small that $c_{7} \bar{\xi}^{\Delta(t)} \leq \frac{1}{2}$. That is, $\bar{\xi} \leq\left(2 c_{7}\right)^{-\frac{1}{\Delta(t)}}$.

Lemma 10. Fix $t \geq t^{*}$ as above, and consider a time-homogeneous discrete time Markov chain, $Z$, with state space $\{1,2, \ldots, r+1\}$, and one-step transition probabilities

$$
p_{i j}= \begin{cases}c_{7} \rho^{\Delta_{i}(t)} & \text { if } j=i+1 \\ 1-c_{7} \rho^{\Delta_{i}(t)} & \text { if } j=i-1 \\ 0 & \text { otherwise, }\end{cases}
$$

for $2 \leq i \leq r, c_{7}$ as defined in Lemma 8, and $0<\rho<1$. Assume that states 1 and $r+1$ are absorbing. Define $q_{i}$ for $2 \leq i \leq r$ by

$$
q_{i}=P\left\{Z \text { hits } r+1 \text { before it hits } i-1 \mid Z_{0}=i\right\} .
$$

Then, for any $\rho \leq \bar{\xi}$,

$$
q_{2} \leq\left(2 c_{7}\right)^{r-1} \rho^{\left(f_{r+1}-f_{2}+\lambda_{t}\left(p_{r+1}-p_{2}\right)\right)} .
$$


We can now bound the probability that, starting one level above the bottom, the $(Y, T)$ process escapes the cup before hitting the cup bottom.

Lemma 11. Fix $\bar{t} \geq t^{*}$ so that $\eta_{\bar{t}} \leq \bar{\xi}$ and suppose that $\bar{x} \in \Pi_{2}$. Then, under the conditions of Theorem 3 , $P\{Y$ visits $F$ before visiting $B \mid(Y(0), T(0))=(\bar{x}, \bar{t})\} \leq\left(2 c_{7}\right)^{r-1} \eta_{\bar{t}}\left(f_{r+1}-f_{2}+\lambda_{\bar{t}}\left(p_{r+1}-p_{2}\right)\right)$

Proof. Define $V_{0}=0$ and

$$
V_{i+1}=\inf \left\{n \geq V_{i}: Y(n) \in D \text { and }(f(Y(n)), p(Y(n))) \neq\left(f\left(Y\left(V_{i}\right), p\left(Y\left(V_{i}\right)\right)\right)\right\} \wedge W .\right.
$$

Note that $V_{i}$ is the iteration that the process changes levels within the set $D$ for the $i^{\text {th }}$ time. We focus on $\left(V_{i}: i \geq 0\right)$ since the process can only escape the cup or hit the bottom through a state in $D$.

Define a function $\zeta$ on $\mathcal{S}$ such that for $x \in L\left(f_{i}, p_{i}\right), \zeta(x)=i$. Consider the process $\left(\zeta\left(Y\left(V_{i}\right)\right): i \geq 0\right)$. For example, $\zeta\left(Y\left(V_{0}\right)\right)=\zeta(\bar{x})=2$. Using Lemma 9 , we determine that

$$
P\left\{\zeta\left(Y\left(V_{i+1}\right)\right)=j+1 \mid \zeta\left(Y\left(V_{i}\right)\right)=j, T\left(V_{i}\right)=\bar{t}\right\} \leq c_{7} \eta\left(\Delta_{j}(\bar{t})\right) .
$$

Setting $\rho \geq \eta_{\bar{t}}$ in Lemma 10, we see that $\left(Z_{k}: k \geq 0\right)$ stochastically dominates $\left(\zeta\left(Y\left(V_{i}\right)\right): i \geq 0\right)$. We conclude that $\left(\zeta\left(Y\left(V_{i}\right)\right): i \geq 0\right)$ has a smaller chance of escaping the cup before hitting the bottom than $\left(Z_{i}, i \geq 0\right)$.

Proof of Theorem 3. Assume that $Y(0)=x_{0} \in B$ and $T(0)=t_{0} \geq t^{*}$. All probabilities and expectations in this proof are defined with respect to the probability measure induced by $(Y(0), T(0))=\left(x_{0}, t_{0}\right)$.

Define $S_{0}=0$ and $S_{i+1}=\inf \left\{j>S_{i}: Y(j) \in B\right\} \wedge W$ for $i \geq 0$. Notice that $S_{i}$ is the iteration at which the process returns to $B$ for the $i^{\text {th }}$ time (until eventually escaping the cup). Also, let $N^{*}=\inf \left\{i: Y\left(S_{i}\right) \in F\right\}$ so that $N^{*}$ is the number of times that $Y$ visits $B$ before escaping the cup. Let $\mathcal{G}_{i}$ denote the $\sigma$-algebra generated by $\left(Y(j): 0 \leq j \leq S_{i}, T(0)\right)$.

From Lemma 11, we see that on the event $\left\{Y\left(S_{n}\right) \in B\right\}$,

$$
\begin{aligned}
P\left\{N^{*}=n+1 \mid \mathcal{G}_{n}\right\} & \leq \eta\left(\Delta_{1}\left(T\left(S_{n}\right)\right)\right)\left(2 c_{7}\right)^{r-1} \eta\left(\left(f_{r+1}-f_{2}+\lambda_{T\left(S_{n}+1\right)}\left(p_{r+1}-p_{2}\right)\right)\right) \\
& \leq\left(2 c_{7}\right)^{r-1} \eta\left(d_{T\left(S_{n}\right)}\right) .
\end{aligned}
$$

Since the event $\left\{N^{*}>n\right\}$ can be determined from the $\sigma$-algebra $\mathcal{G}_{n}$, we have that

$$
\begin{aligned}
P\left\{N^{*}>n+1 \mid \mathcal{G}_{n}\right\} & =\left(1-P\left\{N^{*}=n+1 \mid \mathcal{G}_{n}\right\}\right) I\left\{N^{*}>n\right\} \\
& \geq\left(1-\left(2 c_{7}\right)^{r-1} \eta\left(d_{T\left(S_{n}\right)}\right)\right) I\left\{N^{*}>n\right\} \\
& \geq\left(1-\left(2 c_{7}\right)^{r-1} \eta\left(d_{t_{0}+n}\right)\right) I\left\{N^{*}>n\right\}
\end{aligned}
$$

where the last inequality holds since the bottom of any cup is composed entirely of "real" states implying that $T\left(S_{n}\right) \geq T(0)+n$. Taking expectations,

$$
P\left\{N^{*}>n+1\right\} \geq\left(1-\left(2 c_{7}\right)^{r-1} \eta\left(d_{t_{0}+n}\right)\right) P\left\{N^{*}>n .\right\}
$$


Applying (10) recursively (and assuming that $\eta\left(d_{t_{0}}\right)<\left(2 c_{7}\right)^{-(r-1)}$ so that each term is positive), we get

$$
P\left\{N^{*}>n+1\right\} \geq \prod_{l=0}^{n}\left(1-\left(2 c_{7}\right)^{r-1} \eta\left(d_{t_{0}+l}\right)\right) .
$$

Suppose that $\bar{\xi}$ is defined sufficiently small so that $\eta\left(d_{t_{0}}\right) \leq \bar{\xi}$ implies $\left(2 c_{7}\right)^{r-1} \eta\left(d_{t_{0}}\right) \leq x$ where $x$ is the minimal positive solution to $1-x=\exp (-2 x)$. For such $\eta\left(d_{t_{0}}\right), 1-\left(2 c_{7}\right)^{r-1} \eta\left(d_{t_{0}}\right) \geq \exp \left(-2\left(2 c_{7}\right)^{r-1} \eta\left(d_{t_{0}}\right)\right)$. With this assumption on $\eta\left(d_{t_{0}}\right)$ and the observation that $\left\{N^{*}>q\right\} \subset\{Y(k) \in C$ for $0 \leq k \leq q\}$, we obtain

$$
\begin{aligned}
P\{Y(k) \in C \text { for } 0 \leq k \leq q\} & \geq P\left\{N^{*}>q\right\} \\
& \geq \prod_{k=0}^{q-1}\left(1-\left(2 c_{7}\right)^{r-1} \eta\left(d_{t_{0}+k}\right)\right) \\
& \geq \prod_{k=0}^{q-1} \exp \left(-2\left(2 c_{7}\right)^{r-1} \eta\left(d_{t_{0}+k}\right)\right) \\
& =\exp \left(-2\left(2 c_{7}\right)^{r-1} \sum_{k=0}^{q-1} \eta\left(d_{t_{0}+k}\right)\right) \\
& \geq \exp \left(-2\left(2 c_{7}\right)^{r-1} \sum_{k=0}^{q-1} \eta\left(d_{T(k)}\right)\right) .
\end{aligned}
$$

By setting $c_{6} \geq 2\left(2 c_{7}\right)^{r-1}$ and $\bar{\xi} \leq \min \left\{\left(2 c_{7}\right)^{-\frac{1}{\Delta\left(t^{*}\right)}},(.79681213)\left(2 c_{7}\right)^{-(r-1)}\right\}$, Theorem 3 holds for an arbitrary cup $C$. Repeating for all other cups, we can choose $\bar{\xi}$ and $c_{6}$ to depend only on $\Theta$ and obtain the desired result.

\section{Settling into Deep Cups}

In this section, we present a final theorem that helps complete the proof of Theorem 1. For fixed $(S, P)$, define $E_{S, P}$ as the set of local minimum with depth pairs greater than $(S, P)$. Formally stated, $E_{S, P}=\{x \mid x$ is a local minimum such that $(S(x), P(x)) \succ(S, P)\}$. Additionally, define $O_{S, P}=\{y \mid y$ is accessible from some $x \in E_{S, P}$ at level $\left(f^{\prime}=S+f(x), p^{\prime}=P+p(x)\right)$.

Theorem 4. Let $(S, P)$ be given, and consider a cup $C$ with depth pair $(S, P)$, so that $d_{T(k)}=S+\lambda_{T(k)} P$. If $\sum_{k=0}^{\infty} \eta\left(S+\lambda_{T(k)} P\right)=+\infty$, then $\lim _{k \rightarrow \infty} P\left\{Y(k) \in O_{S, P}\right\}=1$.

To prove Theorem 4, we partition the state space $\mathcal{S}$ into the sets $\left\{U, C_{1}, \ldots, C_{l}\right\}$ so that $U=$ $\left\{z \in \mathcal{S}: E_{S, P}\right.$ is accessible at $(f(z), p(z))$ from $\left.z\right\}$, and $\left\{C_{1}, \ldots, C_{l}\right\}$ are cups with $\left(S\left(C_{i}\right), P\left(C_{i}\right)\right) \prec(S, P)$ for all $i=1, \ldots, l$. Figure 6 illustrates our new perspective of the solution space.

We will invoke the results of Theorems 2 and 3 to prove Theorem 4. Set $\xi$ equal to the minimum of $\bar{\xi}$ as in Theorem 3 and $\min _{i=1, \ldots, l}\left\{\xi_{i}\right\}$ as in Theorem 2, where $l$ indexes the (finite number of) cups.

Let $A_{0}=0$ and $A_{i+1}=\inf \left\{k>A_{i}: Y(k) \in U\right\}$ for $i \geq 0$. Then $A_{i}$ denotes the iteration when the process is in $U$ for the $i^{\text {th }}$ time. Further, define $\alpha=\inf \left\{i: Y\left(A_{i}\right) \in E_{S, P}\right\}$ and $\beta=\inf \left\{i \geq \alpha: Y\left(A_{i}\right) \notin O_{S, P}\right\}$. Note 


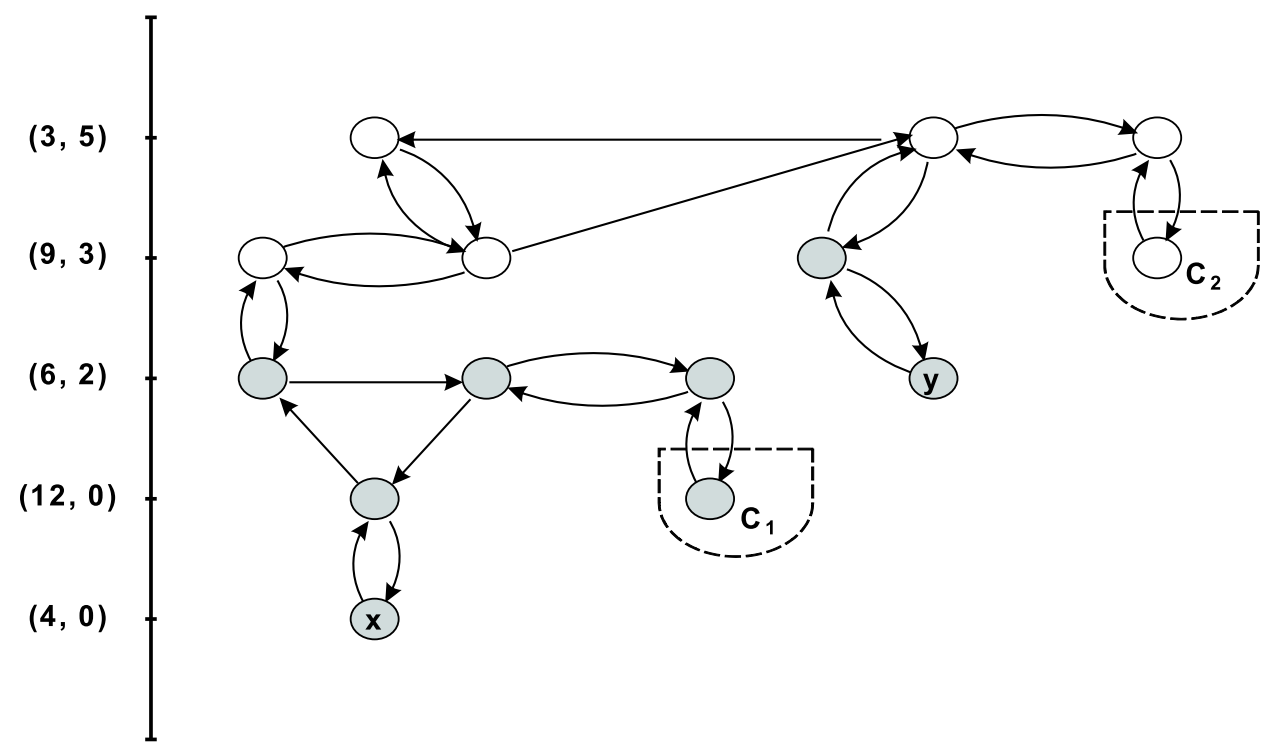

Figure 6: Let $(S, P)=(-4,3)$. This diagram illustrates the partition of $\mathcal{S}$ into $U$ and a set of cups, $\left\{C_{1}, \ldots, C_{l}\right\}$, such that $\left(S\left(C_{i}\right), P\left(C_{i}\right)\right) \prec(-4,3)$ for $i=1, \ldots, l$. Cups are enclosed with dashed lines, and all other states are in $U$. The shaded nodes represent states in the set $O_{(-4,3)}$, and $x$ and $y$ compose $E_{(-4,3)}$.

that $\alpha$ is the number of times that $Y(k)$ visits $U$ by the time it visits the set $E_{S, P}$ for the first time. The related term $\beta$ is the number of times that $Y(k)$ visits $U$ between first visiting $E_{S, P}$ and climbing to a state $z \notin O_{S, P}$.

We now state three lemmas that are proved using the results of Theorem 2. In Lemma 12, we bound the expected accumulated jump probabilities until escaping a cup $C_{i}$. Lemma 13 shows that the expected number of times that $Y$ hits the set $U$ before hitting the bottom of a cup with a depth pair greater than $(S, P)$ is finite. The results of Lemmas 12 and 13 are then combined to bound the expected accumulated jump probabilities until hitting a state in $E_{S, P}$.

Unless noted otherwise, we define all probabilities and expectations with respect to the probability measure induced when $(Y(0), T(0))=\left(x_{0}, t_{0}\right)$ for $x_{0} \in \mathcal{S}$ and $t_{0} \geq t^{*}$.

Lemma 12. Under the conditions of Theorem 2, there exists $c_{8}>0$ depending only on $\Theta$ such that if $T\left(A_{i}+1\right) \geq t_{0}$ and $\eta\left(r_{t_{0}}\right) \leq \xi$, then

$$
E\left[\sum_{k=A_{i}+1}^{A_{i+1}} \eta\left(S+\lambda_{T(k)} P\right) \mid Y\left(A_{i}+1\right), T\left(A_{i}+1\right)\right] \leq c_{8}
$$

Proof. There are two cases to consider, $Y\left(A_{i}+1\right)=Y\left(A_{i+1}\right) \in U$ or $Y\left(A_{i}+1\right) \notin U$. The result follows directly from Theorem 2(a) and the fact that $Y$ cannot jump directly from one of the cups to another without visiting a state in $U$. 
Lemma 13. Under the conditions of Theorem 2, there exists a constant $c_{9}>0$ depending only on $\Theta$ such that $E[\alpha] \leq c_{9}$.

Proof. For each $x \in U$, there exists $n=n(x)$, and a sequence of distinct states, $\left\{x=s_{0}, \ldots, s_{n}=z\right\}$, such that $s_{j} \in U$ for $0 \leq j \leq n,\left(f\left(s_{0}\right), p\left(s_{0}\right)\right) \succeq\left(f\left(s_{1}\right), p\left(s_{1}\right)\right) \succeq \cdots\left(f\left(s_{n}\right), p\left(s_{n}\right)\right)$, and $s_{n} \in E_{S, P}$. Along this path, there are two ways that the process can move from $s_{j}$ to $s_{j+1}$, either (i) $Y$ moves from $s_{j}$ to $s_{j+1}$ by direct transition or (ii) $Y$ descends into a cup and climbs out via $s_{j+1}$. By Theorem 2(b), we see that the probabilities of such (potentially multistep) transitions are bounded below. Therefore, a standard geometric trials argument shows that $\alpha$ has a geometrically decaying tail and so $E[\alpha] \leq c_{9}$.

Lemma 14. Under the conditions of Theorem 2, there is a constant $c_{10}$ depending only on $\Theta$ such that for every $x_{0} \in \mathcal{S}$, and every $t_{0} \geq t^{*}$ with $\eta\left(r_{t_{0}}\right) \leq \xi$,

$$
E\left[\sum_{k=0}^{A_{\alpha}} \eta\left(d_{T(k)}\right)\right] \leq c_{10} .
$$

Proof. The proof is similar to that of Lemma 4, and uses Lemma 12 and Lemma 13.

We need one more preparatory lemma before proving the main result of this section.

Lemma 15. For $T(0)=t_{0}, \sum_{k=0}^{\infty} \eta\left(d_{T(k)}\right)=\infty$ if and only if $\sum_{k=0}^{\infty} \eta\left(d_{t_{0}+k}\right)=\infty$.

Proof. First suppose $\sum_{k=0}^{\infty} \eta\left(d_{T(k)}\right)=\infty$. Note that the most consecutive artificial states that one may visit is $\ell-1$ since any state in $\left(f_{1}, p_{1}\right)$ and $\left(f_{\ell}, p_{\ell}\right)$ must be real. Therefore, the clock will be paused for at most $\ell-1$ consecutive iterations at a time, and so

$$
(\ell-1)\left[\sum_{k=0}^{\infty} \eta\left(d_{t_{0}+k}\right)\right] \geq \sum_{k=0}^{\infty} \eta\left(d_{T(k)}\right)=\infty .
$$

The converse is immediate since $T(k) \leq t_{0}+k$, and so $\eta_{T(k)} \geq \eta_{t_{0}+k}$.

Proof of Theorem 4. Unless specified otherwise, all probabilities and expectations in this proof are defined with respect to the probability measure induced when $(Y(0), T(0))=\left(x_{0}, t_{0}\right)$ for $x_{0} \in \mathcal{S}$ and $t_{0} \geq t^{*}$. Notice that

$$
\begin{aligned}
P\left\{A_{\alpha}>q\right\} \sum_{l=0}^{q} \eta\left(d_{t_{0}+l}\right) & =E\left[I\left\{q<A_{\alpha}\right\} \sum_{l=0}^{q} \eta\left(d_{T(0)+l}\right)\right] \\
& \leq E\left[\sum_{l=0}^{A_{\alpha}} \eta\left(d_{T(0)+l}\right)\right] \\
& \leq E\left[\sum_{l=0}^{A_{\alpha}} \eta\left(d_{T(l)}\right)\right] \\
& \leq c_{10} .
\end{aligned}
$$

Thus,

$$
P\left\{A_{\alpha} \leq q\right\} \geq 1-\frac{c_{10}}{\sum_{l=0}^{q} \eta\left(d_{t_{0}+l}\right)} .
$$


Recognize that $Y\left(A_{\alpha}\right)$ must be in the bottom of a cup $\bar{C}$ with depth pair $(S(\bar{C}), P(\bar{C})) \succeq\left(S+\gamma_{S}, P+\gamma_{P}\right)$ where

$$
\begin{aligned}
& \left(\gamma_{S}, \gamma_{P}\right)= \\
& \quad \min \left\{\left(S\left(C^{\prime}\right)-S, P\left(C^{\prime}\right)-P\right): C^{\prime} \text { is a cup with depth pair }\left(S\left(C^{\prime}\right), P\left(C^{\prime}\right)\right) \succ(S, P)\right\} .
\end{aligned}
$$

We can relate the dynamic depth of $\bar{C}$ at iteration $k$ to $(S, P)$ by

$$
\begin{aligned}
\bar{d}_{T(k)} & =S(\bar{C})+\lambda_{T(k)} P(\bar{C}) \\
& \geq S+\lambda_{T(k)} P+\gamma_{S}+\lambda_{T(k)} \gamma_{P} \\
& =d_{T(k)}+\gamma_{S}+\lambda_{T(k)} \gamma_{P} .
\end{aligned}
$$

Conditioning on $A_{\alpha}$ and applying Theorem 3, we find that

$$
\begin{aligned}
P\left\{A_{\beta}>q\right\} & \geq \exp \left(-c_{6} \sum_{k=0}^{q-1} \eta\left(\bar{d}_{T(k)}\right)\right) \\
& =\exp \left(-c_{6} c_{11} \sum_{k=0}^{q-1} \eta\left(\bar{d}_{t_{0}+k}\right)\right) \\
& \geq \exp \left(-c_{6} c_{11} \eta\left(\gamma_{S}+\lambda_{t_{0}} \gamma_{P}\right) \sum_{k=0}^{q} \eta\left(d_{t_{0}+k}\right)\right),
\end{aligned}
$$

for some constant $c_{11} \geq 1$.

Since $Y(q) \in O_{S, P}$ if $A_{\alpha} \leq q<A_{\beta}$, we have

$$
\begin{aligned}
P\left\{Y(q) \in O_{S, P}\right\} & \geq P\left\{A_{\alpha} \leq q<A_{\beta}\right\} \\
& \geq \exp \left(-c_{6} c_{11} \eta\left(\gamma_{S}+\lambda_{t_{0}} \gamma_{P}\right) \sum_{k=0}^{q} \eta\left(d_{t_{0}+k}\right)\right)-\frac{c_{10}}{\sum_{k=0}^{q} \eta\left(d_{t_{0}+k}\right)}
\end{aligned}
$$

where we have used the inequality $P\{A \cap B\} \geq P\{A\}+P\{B\}-1$.

We now show that this inequality gives $\lim _{q \rightarrow \infty} P\left\{Y(q) \in O_{S, P}\right\}=1$. Let $\epsilon>0$ be arbitrary except $\frac{c_{10}}{\epsilon}>1$. Now choose $t_{3}$ such that if $t_{0}>t_{3}$ then

$$
\exp \left(-c_{6} c_{11} \eta\left(\gamma_{S}+\lambda_{t_{0}} \gamma_{P}\right)\left(\frac{3 c_{10}}{\epsilon}\right)\right) \geq 1-\frac{\epsilon}{2}
$$

Choose $q^{*}\left(t_{3}\right)$ such that

$$
\sum_{k=0}^{q^{*}\left(t_{3}\right)} \eta\left(d_{t_{3}+k}\right) \geq \frac{2 c_{10}}{\epsilon}
$$

which is possible by Lemma 15 . Now, for all $q>q^{*}\left(t_{3}\right)$, there exists $t_{1}(q), t_{2}(q)$ such that (i) $t_{3} \leq t_{1}(q)<$ $t_{2}(q)$, (ii) $\frac{2 c_{10}}{\epsilon} \leq \sum_{k=0}^{q} \eta\left(d_{t_{0}+k}\right) \leq \frac{3 c_{10}}{\epsilon}$ for all $t_{0} \in\left[t_{1}(q), t_{2}(q)\right]$, and (iii) $t_{2}(q)-t_{1}(q)>1$. Then, for 
$t_{0} \in\left[t_{1}(q), t_{2}(q)\right]$

$$
\begin{aligned}
P\left\{Y(q) \in O_{S, P}\right\} & \geq \exp \left(-c_{6} c_{11} \eta\left(\gamma_{S}+\lambda_{t_{0}} \gamma_{P}\right) \sum_{k=0}^{q} \eta\left(d_{t_{0}+k}\right)\right)-\frac{c_{10}}{\sum_{k=0}^{q} \eta\left(d_{t_{0}+k}\right)} \\
& \geq \exp \left(-c_{6} c_{11} \eta\left(\gamma_{S}+\lambda_{t_{0}} \gamma_{P}\right)\left(\frac{3 c_{10}}{\epsilon}\right)\right)-\frac{c_{10}}{\sum_{k=0}^{q} \eta\left(d_{t_{0}+k}\right)} \\
& \geq\left(1-\frac{\epsilon}{2}\right)-\frac{\epsilon}{2} \\
& =1-\epsilon .
\end{aligned}
$$

This probability bound is conditioned on $(Y(0), T(0))=\left(x_{0}, t_{0}\right)$ where $t_{0} \in\left[t_{1}(q), t_{2}(q)\right]$. So when $T(0)=0$, let $\rho=\min \left\{k: T(k) \geq t_{1}(q)\right\}$. Since $t_{2}(q)-t_{1}(q)>1, T(\rho)<t_{2}(q)$. Now all probabilities and expectations are induced by $(Y(0), T(0))=\left(x_{0}, 0\right)$. We find that

$$
\begin{aligned}
P\left\{Y(q) \in O_{S, P}\right\} & =E\left[P\left\{Y(q) \in O_{S, P} \mid(Y(\rho), T(\rho))\right\}\right] \\
& \geq E[(1-\epsilon)] \\
& =1-\epsilon .
\end{aligned}
$$

Since $\epsilon$ was arbitrary, the result follows.

\section{$7 \quad$ Proof of Main Result}

In this section, we conclude the proof of Theorem 1 by assembling results from Theorems 3 and 4 . With the help of an additional lemma, we modify the final argument in Hajek (1988) to accommodate the concept of levels.

Lemma 16. If $\sum_{k=0}^{\infty} \eta\left(S+\lambda_{T(k)} P\right)<+\infty$ and $\sum_{k=0}^{\infty} \eta\left(\bar{S}+\lambda_{T(k)} \bar{P}\right)=+\infty$, then $(S, P) \succ(\bar{S}, \bar{P})$.

Proof. We prove by contradiction by assuming $(S, P) \preceq(\bar{S}, \bar{P})$. Then, either (i) $P<\bar{P}$ and nothing can be said about the relative sizes of $S$ and $\bar{S}$, or (ii) $P=\bar{P}$ and $S \leq \bar{S}$. In either case, by our construction of a compression schedule, there exists $k^{*} \geq 0$ such that $\eta\left(S+\lambda_{T(k)} P\right) \geq \eta\left(\bar{S}+\lambda_{T(k)} \bar{P}\right)$ for $k \geq k^{*}$. Therefore,

$$
\begin{aligned}
\sum_{k=0}^{\infty} \eta\left(S+\lambda_{T(k)} P\right) & \geq \sum_{k=k^{*}}^{\infty} \eta\left(S+\lambda_{T(k)} P\right) \\
& \geq \sum_{k=k^{*}}^{\infty} \eta\left(\bar{S}+\lambda_{T(k)} \bar{P}\right) \\
& =\infty
\end{aligned}
$$

which is a contradiction.

Proof of Theorem 1. When $S=0$ and $P=0, O_{S, P}$ is the set of all local minima and the condition of Theorem 4 holds. Thus Theorem 4 implies part (a) of Theorem 1.

We will next prove the "if" half of part(b) of Theorem 1 . Let $B_{S, P}$ denote the bottom of a cup $C$ with depth pair $(S, P)$. Suppose that the states in $B_{S, P}$ are local minima with static depth $S$ and penalty depth $P$, 
i.e., $C$ is the largest cup containing the states in $B_{S, P}$ as local minima. We must show that $P\left\{Y(k) \in B_{S, P}\right\}$ has limit zero as $k$ tends to infinity if $\sum_{k=0}^{\infty} \eta\left(S+\lambda_{T(k)} P\right)=\sum_{k}^{\infty} \eta\left(d_{T(k)}\right)=\infty$. In view of Theorem 4 , it is sufficient to prove that $B_{S, P} \cap O_{S, P}=\emptyset$. For the sake of contradiction, suppose there exists $x \in B_{S, P} \cap O_{S, P}$. Then there is a state $y \in E_{S, P}$ so that (i) $y$ is a local minimum with $(S(y), P(y)) \succ(S, P)$, (ii) $x$ is accessible from $y$ at $(S+f(y), P+p(y))$, and (iii) $y$ is accessible from $x$ at $(S+f(y), P+p(y))$.

Case 1: $(f(x), p(x)) \succ(f(y), p(y))$

Since $x \in B_{S, P},\left(f^{\prime}=S+f(x), p^{\prime}=P+p(x)\right)$ is the lowest level such that a state $z$, with $(f(z), p(z)) \prec$ $(f(x), p(x))$, is accessible from $x$. However, by (iii), we know that $y$ is accessible from $x$ at $(S+f(y), P+$ $p(y))$. This contradicts the fact that $x$ is a local minimum with $(S(x), P(x))=(S, P)$, since $(f(y), p(y)) \prec$ $(f(x), p(x))$, and so $(S+f(y), P+p(y)) \prec(S+f(x), P+p(x))$.

Case 2: $(f(x), p(x)) \preceq(f(y), p(y))$

Since $x \in B_{S, P}$, there exists a state $z$, with $(f(z), p(z)) \prec(f(x), p(x))$, accessible from $x$ at $(S+f(x), P+$ $p(x))$. Since $(f(x), p(x)) \preceq(f(y), p(y)), z$ is also accessible from $x$ at $(S+f(y), P+p(y))$. Combining this observation with (ii), note that $z$ is accessible from $y$ at $(S+f(y), P+p(y))$. This contradicts (i), since $(f(z), p(z)) \prec(f(x), p(x)) \preceq(f(y), p(y))$ and (i) implies that $\left(f^{\prime \prime}, p^{\prime \prime}\right) \succ(S+f(y), P+p(y))$ is the lowest level such that a state $w$, with $(f(w), p(w)) \prec(f(y), p(y))$, is accessible from $y$.

We obtain a contradiction in both cases, so we have proved that $B_{S, P}$ and $O_{S, P}$ are disjoint. This completes the proof of the "if" half of part (b) of Theorem 1.

To prove the "only if" half of part (b) of Theorem 1, we will prove the contrapositive. Again let $B_{S, P}$ denote the bottom of a cup $C$ with depth pair $(S, P)$, and suppose that the states in $B_{S, P}$ are local minima of static depth $S$ and penalty depth $P$. Assume that $\eta_{t}$ and $\lambda_{t}$ are given such that $\sum_{k=0}^{\infty} \eta\left(S+\lambda_{T(k)} P\right)<+\infty$. We want to prove that $P\left\{Y(k) \in B_{S, P}\right\}$ does not converge to zero as $k$ tends to infinity.

Since $\sum_{k=0}^{\infty} \eta\left(S+\lambda_{T(k)} P\right)<+\infty$, we know from Lemma 16 that $(S, P) \succ(\bar{S}, \bar{P})$, where $(\bar{S}, \bar{P})$ are the largest value pair such that $\sum_{k=0}^{\infty} \eta\left(\bar{S}+\lambda_{T(k)} \bar{P}\right)=+\infty$. Select a state $y \in B_{S, P}$ and let $\bar{C}=\{x: x$ is accessible from $y$ at level $(\bar{S}+f(y), \bar{P}+p(y))\}$. Let $\bar{B}$ denote the bottom of cup $\bar{C}$, and let $x_{0} \in \bar{B}$.

The fact that $\sum_{k=0}^{\infty} \eta\left(d_{T(k)}(\bar{C})\right)<+\infty$ (since $\left.(S(\bar{C}), P(\bar{C})) \succ(\bar{S}, \bar{P})\right)$ and Theorem 3 imply that

$$
\lim _{k \rightarrow \infty} \inf P\{Y(k) \in \bar{C}\}>0 .
$$

If $z \in \bar{C}-\bar{B}$, then $z$ is not a local minimum or $z$ is a local minimum with depth pair $(S(z), P(z)) \prec(\bar{S}, \bar{P})$. Using this observation, part (a) of Theorem 1, and the "if" half of part (b) of Theorem 1,

$$
\lim _{k \rightarrow \infty} P\{Y(k)=z\}=0 \text { for } z \in \bar{C}-\bar{B} .
$$

From (11) and (12), we conclude that $\liminf _{k \rightarrow \infty} P\{Y(k) \in \bar{B}\}>0$, and since $\bar{B} \subset B$, this inequality is true with $\bar{B}$ replaced by $B$. This completes the "only if" half of part (b) of Theorem 1 . 


\section{Conclusion}

We have developed a necessary and sufficient condition for the convergence in probability of simulated annealing in the presence of complicating constraints. Through careful construction of the concept of levels, we are able to maintain the monotonicity of the long-run transition probabilities and therefore modify the mechanics in Hajek (1988) to achieve the desired result. We have rigorously established that, to converge to the set of global minima, slower cooling than proposed in Hajek (1988) is required. Therefore, it is unlikely that we can view compressed annealing as a global optimization method, but rather as a heuristic implemented with accelerated cooling and compression schedules.

The results presented in this paper shed light on the the performance of the algorithm, but more work is needed. One future endeavor is to create a class of problems for which we can derive analytical results on probability of convergence and rate of convergence in order to garner further insight on the algorithm's behavior. Future research may include the analysis of annealing behavior during the transient period of compression $\left(\lambda<\lambda^{*}\right)$ and its impact on the quality of heuristic solutions in practical applications.

\section{Acknowledgments}

The work of Jeff Ohlmann and Shane Henderson was partially supported by National Science Foundation Grant Number DMI-0230528.

\section{References}

E.H.L. Aarts and J.H.M. Korst. 1989. Simulated Annealing and Boltzmann machines. John Wiley and Sons, Chichester.

S. Anily and A. Federgruen. 1987 Simulated annealing methods with general acceptance probabilities. Journal of Applied Probability 35 657-667.

V. Cerny. 1985. A thermodynamical approach to the travelling salesman problem: An efficient simulation algorithm. Journal of Optimization Theory and Applications 45 41-55.

T.S. Chiang and Y. Chow. 1988. On the convergence rate of annealing processes. SIAM Journal on Control and Optimization 26 1455-1470.

A. Frigerio and G. Grillo. 1993. Simulated annealing with time-dependent energy function. Mathematische Zeitschrift $21397-116$.

D. Geman. 1990. Random fields and inverse problems in imaging. P. Hennequin, ed., Lecture Notes in Mathematics. Springer-Verlag, 117-193.

S. Geman and D. Geman. 1984. Stochastic relaxation, gibbs distributions, and the bayesian restoration of images. IEEE Transactions on Pattern Analysis and Machine Intelligence 6 721-741.

B. Gidas. 1985. Nonstationary markov chains and convergence of the annealing algorithm. Journal of Statistical Physics 39 73-131.

A. Hadj-Alouane and J. Bean. 1997. A genetic algorithm for the multiple-choice integer program. Operations Research 45 92-101.

B. Hajek. 1988. Cooling schedules for optimal annealing. Mathematics of Operations Research 13 311-329. 
R. Holley and D. Stroock. 1988. Simulated annealing via sobolev inequalities. Communications in Mathematical Physics 115 553-569.

A.W. Johnson and S.H. Jacobson. 2002. On the convergence of generalized hill climbing algorithms. Discrete Applied Mathematics 119 37-57.

S. Kirkpatrick, C.D. Gellat, and M.P. Vecchi. 1983. Optimization by simulated annealing. Science, 220 671-680.

M. Lundy and A. Mees. 1986. Convergence of an annealing algorithm. Mathematical Programming 34 111-124.

P. Del Moral and L. Miclo. 1999. On the convergence and applications of generalized simulated annealing. SIAM Journal on Control and Optimization 37 1222-1250.

N. Metropolis, A.W. Rosenbluth, M.N. Rosenbluth, A.H. Teller, and E. Teller. 1953. Equation of state calculation by fast computing machines. Journal of Chemical Physics 21 1087-1091.

D. Mitra, F. Romeo, and A.L. Sangiovanni-Vincentelli. 1986. Convergence and finite-time behavior of simulated annealing. Advances in Applied Probability 18 747-771.

C. Morse. 1997. Stochastic Equipment Replacement with Budget Constraints. PhD thesis, University of Michigan, Ann Arbor.

C.R. Reeves, editor. 1993. Modern Heuristic Techniques for Combinatorial Problems. John Wiley and Sons, New York.

M.C. Robini, Y. Bresler, and I.E. Magnin. 2002. On the convergence of metropolis-type relaxation and annealing with constraints. Probability in the Engineering and Informational Sciences 16 427-452.

V.E. Theodoracatos and J.L. Grimsley. 1995. The optimal packing of arbitrarily-shaped polygons using simulated annealing and polynomial-time cooling schedules. Computer Methods in Applied Mechanics and Engineering $\mathbf{1 2 5}$ $53-70$.

J. Yao. 2000. On constrained simulation and optimization by metropolis chains. Statistics and Probability Letters $\mathbf{4 6}$ 187-193. 